\title{
Model-based inference of neutralizing antibody avidities against influenza virus
}

\author{
Janina Linnik ${ }^{1,2}$, Mohammedyaseen Syedbasha ${ }^{3}$, Yvonne Hollenstein ${ }^{3}$, \\ Jörg Halter ${ }^{4}$, Adrian Egli ${ }^{3,5, *}$, Jörg Stelling ${ }^{1,2, *}$ \\ ${ }^{1}$ Department of Biosystems Science and Engineering, ETH Zurich, Basel, \\ Switzerland \\ ${ }^{2}$ Swiss Institute for Bioinformatics, Basel, Switzerland \\ ${ }^{3}$ Department of Biomedicine, University of Basel, Basel, Switzerland \\ ${ }^{4}$ Division of Hematology, University Hospital of Basel, Basel, Switzerland \\ ${ }^{5}$ Division of Clinical Bacteriology and Mycology, University Hospital of Basel, \\ Basel, Switzerland \\ *Correspondence to: adrian.egli@usb.ch or joerg.stelling@bsse.ethz.ch
}

To assess the response to vaccination, quantity (concentration) and quality (avidity) of neutralizing antibodies are the most important parameters. Specifically, an increase in avidity indicates germinal center formation, which is required for establishing long-term protection. For influenza, the classical hemagglutination inhibition (HI) assay, however, quantifies a combination of both and to separately determine avidity requires high experimental effort. Here, we present a biophysical model that infers neutralizing IgG avidities from HI titers and IgG concentrations. We applied our model to infer IgG avidities against the pandemic 2009 H1N1 influenza virus in vaccinated hematopoietic stem cell transplantation patients $(n=45)$ and validated our results with independent avidity measurements. The model predicted that increased IgG concentrations mainly contribute to observed HI titer increases and that immunosuppressive treatment is associated with lower baseline avidities. Because the model requires only easy-to-establish measurements as inputs, we anticipate that it will help to disentangle causes for poor vaccination outcomes also in larger vaccine studies.

\section{Introduction}

To prevent influenza epidemics, it is crucial to annually develop effective vaccines against circulating influenza strains. To assess vaccine efficacy, hemagglutination inhibition (HI) titers are traditionally used as a surrogate for the influenza-neutralization capacity of vaccine-induced 
antibodies in serum (Palache et al., 2003; Benoit et al., 2015). The HI assay makes use of the phenomenon that influenza viruses can bind with its surface receptor hemagglutinin (HA) to red blood cells (RBCs) and thereby cross-link RBCs to macroscopic cell aggregates in a process called hemagglutination (Hirst, 1941). In the presence of influenza-binding antibodies that block RBC binding sites, hemagglutination is inhibited. This allows quantifying the neutralization capacity of serum antibodies: the highest serum dilution that fully inhibits hemagglutination is determined, and the reciprocal of this dilution is reported as the HI titer (WHO, 2002).

The HI titer measures a combination of both antibody concentration and avidity, where avidity quantifies the overall strength of a multivalent antibody binding to hemagglutinin epitopes involved in virus-RBC interaction (neutralizing binding). When assessing vaccine response, however, it is important to distinguish between antibody concentration and avidity. In particular, no increase in avidity following vaccination indicates a hampered formation of germinal centers (GCs) where B cells undergo affinity maturation and proliferate to memory B cells or to long-lived B cells, providing long-term protection (Berek et al., 1991; Shlomchik and Weisel, 2012; De Silva and Klein, 2015).

Unfortunately, avidity measurements of serum antibodies are time-consuming and costly. In general, these measurements require long incubation times because antibodies dissociate slowly from their respective antigens. Commonly used techniques are surface plasmon resonance (SPR) and elution experiments with chaotropic agents (such as urea) based on enzyme-linked immunosorbent assays (ELISAs). While SPR experiments require special equipment and long calibration, elution assays are very sensitive to experimental conditions, and the optimal protocol might vary substantially for different antigens or even serum samples (Underwood, 1993; Olsson et al., 2019).

In comparison, measurements of $\mathrm{HI}$ titers and influenza-specific serum IgG concentrations are faster to establish and simpler to perform (Kaufmann et al., 2017). HI assays are considered the gold standard and routinely performed in vaccine studies; they proved to be fast, cheap, and reliable. IgG concentrations can be determined in standardized ELISA experiments. These are suitable for large-scale serological studies because they can be fully automated and yield highly reproducible results. Therefore, estimation of avidities from $\mathrm{HI}$ titers and $\mathrm{IgG}$ concentrations would facilitate influenza vaccine studies in larger populations.

Here, we present a biophysical model of the $\mathrm{HI}$ assay that mechanistically describes the relationship of IgG concentration and avidity to $\mathrm{HI}$ titer. The model enables the inference of neutralizing IgG avidities from $\mathrm{HI}$ titers and ELISA-detected $\mathrm{IgG}$ concentrations. We applied it to vaccinated hematopoietic stem cell transplantation (HSCT) patients, focusing on IgG antibodies specific to pandemic influenza A/California/7/2009 (H1N1pmd09). Despite available vaccines, the case fatality rate for influenza infection is 17-29\% in these patients (Kunisaki and Janoff, 2009). Because HSCT patients are commonly immunocompromised due to the post-transplant immune reconstitution and immunosuppressive treatment against graft rejection, and patients with low 
antibody avidities are likely to be at high risk for fatal infections, we investigated the association of inferred avidities with three indicators of immunocompromised status, namely first two years post transplantation, intake of immunosuppressive drugs, and chronic graft-versus-host disease (cGVHD) (Brunette et al., 2015). Our model detected that immunosuppressive treatment is associated with lower baseline avidities, but we did not detect a significant association with cGVHD or the time after transplantation. In addition, our model suggests that vaccination induced affinity maturation of neutralizing antibodies in only a few patients.

\section{Results}

\section{Model of the hemagglutination inhibition (HI) assay}

We combined and extended existing models of antibody-virus interaction (Groth, 1963) and cell-cell agglutination (Dolgosheina et al., 1992) to a model that mechanistically captures the key processes of the HI assay (Figure 1). The HI assay is performed in three consecutive steps (WHO, 2002): (i) Serial dilution of patient serum and $30 \mathrm{~min}$ incubation with influenza virus, (ii) addition of RBCs followed by 30 min incubation, and (iii) determination of the HI titer based on the presence or absence of hemagglutination inhibition in each serum dilution (Figure 1, top). We represent these steps separately: the model output of one step serves as input for subsequent steps (Figure 1, bottom).

Step 1 (binding of antibodies to virus): We modeled the binding of $\mathrm{IgG}$ antibodies to virus hemagglutinin (HA) as a diffusion-controlled reversible reaction between IgG molecules and virus particles (see Methods for all details). Each homotrimeric HA receptor has three identical binding sites for monoclonal IgG, but we assume that one HA trimer accommodates at most one IgG molecule due to steric hindrance (Taylor et al., 1987; Poumbourios et al., 1990; Otterstrom et al., 2014). Serum contains a mixture of polyclonal IgG antibodies. Thus, after the addition of influenza virus to serum, HA-specific IgG clones form a mixture of IgG-HA complexes according to their individual dissociation constants (avidities). We assume that any other interactions are negligible because serum samples are pretreated with proteases to limit unspecific binding. Without information on the individual concentrations or avidities of each IgG clone, we consider the total concentration of HA-specific IgG and the apparent dissociation constant $K_{\mathrm{D}}^{\mathrm{app}}$. This constant is proportional to the ratio of free HA-specific IgG molecules over all formed IgG-HA complexes at equilibrium. Importantly, the inverse $1 / K_{\mathrm{D}}^{\text {app }}$ can be interpreted as the apparent avidity of HA-specific IgG in serum. We compute the fraction of antibody-bound virus at binding equilibrium for each serum dilution (Figure 1, left) as input for step 2 because 30 min incubation suffices to reach binding equilibrium.

Step 2 (hemagglutination): When RBCs are added, virus particles bind reversibly with free HA binding sites to sialic acid (SA) linked receptors on RBCs. We assume that IgG antibodies and SA-linked receptors do not compete for HA binding sites because the affinity of SA 


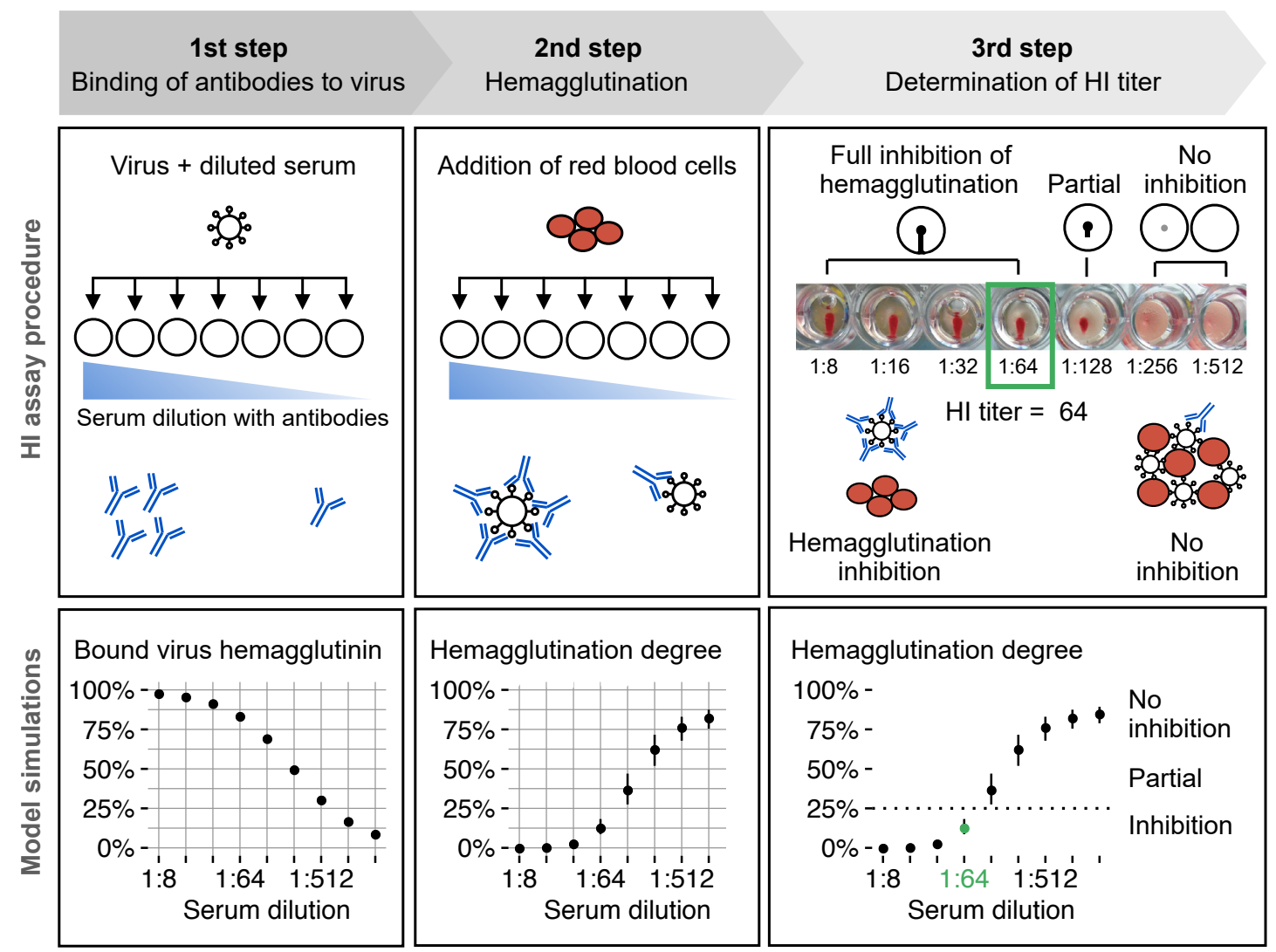

Figure 1. Overview of the hemagglutination inhibition (HI) assay (top) and illustrative simulation results (bottom). First step: patient serum is serially diluted and incubated with a constant amount of influenza virus. The model computes the amount of antibody-bound viral hemagglutinin (HA) for each serum dilution. Second step: red blood cells (RBCs) are added to each dilution and virus particles with free HA binding sites cross-link RBCs to cell aggregates. The model predicts a switch-like increase in agglutinated RBCs with decreasing antibody concentration. Thirds step: the plate is tilted by 90 degrees to detect full hemagglutination inhibition. If none/few RBCs are agglutinated, sedimented RBCs flow down to the rim. By definition, those wells show full hemagglutination inhibition. The reciprocal of the maximal inhibitory dilution is the HI titer. We classify our simulation results into inhibition and no inhibition by setting a threshold at $25 \%$ hemagglutination. Simulation results show median and interquartile range indicating the uncertainty due to experimental conditions (RBC concentration, virus concentration, readout time) and model parameters (summarized in Table 1) for an $\mathrm{IgG}$ serum concentration of $25 \mathrm{nM}(4 \mu \mathrm{g} / \mathrm{mL})$ and $K_{\mathrm{D}}^{\mathrm{app}}=0.1 \mathrm{nM}$. 
to HA is in the mM range (Sauter et al., 1989, 1992; Weinhold and Knowles, 1992), far below the affinity of HA-specific IgGs in the $\mathrm{nM}$ range (Lee et al., 2016). The tight binding of the virus to RBCs results from binding multiple SA moieties simultaneously (Takemoto et al., 1996). The virus-RBC interactions will eventually induce hemagglutination. We model it as a coagulation process (Von Smoluchowski, 1917), where RBCs stick together whenever they collide such that virus particles can cross-link them. Only when a free SA-linked receptor on an RBC meets a free HA on a virus particle that is simultaneously bound to another RBC, the collision leads to a successful cross-link. We define a degree of hemagglutination that takes the value $0 \%$ without any hemagglutination (not a single cross-link), and $100 \%$ when all RBCs form a single aggregate. The model predicts a switch-like increase in the degree of hemagglutination with decreasing antibody concentration, consistent with the experimentally observed switch-like behavior of the assay (Figure 1, middle).

Step 3 (determination of HI titer): After another 30 min incubation, each serum dilution is inspected for hemagglutination inhibition, and the reciprocal of the maximal dilution that shows full inhibition is the HI titer (Figure 1, right). To model this binary decision (inhibition or no inhibition), we classify the outcome by setting a threshold at $25 \%$ hemagglutination because we define 50\% hemagglutination as partial inhibition and our model predicts for $\geq 1$ HA unit virus a hemagglutination degree of $\geq 75 \%$. By definition, this is interpreted as full inhibition (Supplementary Figure S1), suggesting that differences in hemagglutination degree below $25 \%$ or above $75 \%$ cannot be distinguished by eye.

To make the model specific for influenza H1N1pdm09, we extracted parameters from literature for IgG antibodies, the virus strain, and chicken RBCs (Table 1). In addition, we established the agglutination rate parameter from experiments with reference serum (see Methods). The model thereby establishes a quantitative relationship between neutralizing IgG concentration, $\mathrm{HI}$ titer, and avidity. Here, avidity is defined as the inverse of the apparent dissociation constant of the IgG-hemagglutinin complex, $1 / K_{\mathrm{D}}^{\text {app }}$ : the lower the $K_{\mathrm{D}}^{\text {app }}$ value, the tighter the binding and thus the higher the avidity.

\section{The model quantitatively relates IgG concentration, avidity, and HI titer}

To infer antibody avidities accurately, the model needs to be sensitive to the experimental data used as inputs. However, it should not be sensitive to other experimental factors and uncertainties in model parameters. To evaluate the model in this respect, we used Sobol sensitivity analysis (Saltelli et al., 2004), which attributes variance in model output (here: hemagglutination degree) to the individual model input factors. The more influential the input factor is, the higher is its contribution to the variance in hemagglutination degree. We considered the ranges for all model input factors summarized in Table 1. In particular, for IgG concentration and avidity the ranges match the experimentally observed ranges for H1N1pmd09-specific IgG after vaccination in adults, which are approximately $0-420 \mu \mathrm{g} / \mathrm{mL}$ serum IgG (Eidem et al., 
Table 1. Model parameters and variables. Abbreviations are: IgG, Immunoglobulin G; RBC, red blood cell; HA, hemagglutinin; HAU, HA unit; SA, sialic acid.

\begin{tabular}{|c|c|c|c|c|}
\hline Description & Symbol & Value & $\begin{array}{l}\text { Distribution in } \\
\text { sensitivity analysis }\end{array}$ & Reference \\
\hline $\begin{array}{l}\text { Serum IgG } \\
\text { concentration }\end{array}$ & $A_{0}$ & $\begin{array}{l}\text { Sample- } \\
\text { specific }\end{array}$ & $\begin{array}{l}\text { Unif }(0,2800) \mathrm{nM}(0- \\
420 \mu \mathrm{g} / \mathrm{mL})\end{array}$ & Eidem et al. (2015) \\
\hline $\begin{array}{l}\text { Apparent IgG } \\
\text { dissociation } \\
\text { constant }\end{array}$ & $K_{D}^{\mathrm{app}}$ & $\begin{array}{l}\text { Sample- } \\
\text { specific }\end{array}$ & Unif $(0.001,300) \mathrm{nM}$ & Lee et al. (2016) \\
\hline $\begin{array}{l}\text { Initial virus } \\
\text { concentration }\end{array}$ & $V_{0}$ & $\begin{array}{l}1.3 \cdot 10^{-4} \\
\mathrm{nM}(4 \mathrm{HAU})\end{array}$ & $\begin{array}{l}\text { Unif }\left(0.9 \cdot 10^{-4}, 2.3 \cdot 10^{-4}\right) \\
\mathrm{nM}(3-7 \text { HAU })\end{array}$ & WHO (2002) \\
\hline $\begin{array}{l}\text { Initial RBC } \\
\text { concentration }\end{array}$ & $R B C_{0}$ & $\begin{array}{l}3.1 \cdot 10^{-5} \\
\mathrm{nM}\end{array}$ & $\begin{array}{l}\operatorname{Unif}\left(1.6 \cdot 10^{-5}, 6.3 \cdot 10^{-5}\right) \\
\mathrm{nM}\end{array}$ & WHO (2002) \\
\hline $\begin{array}{l}\text { Number of HA } \\
\text { receptors per virus }\end{array}$ & $r$ & 400 & Discrete Unif $(300,500)$ & $\begin{array}{l}\text { Ruigrok et al. } \\
\text { (1984); Harris et al. } \\
(2013)\end{array}$ \\
\hline $\begin{array}{l}\text { Number of epitopes } \\
\text { per HA receptor }\end{array}$ & $e$ & 3 & Fixed at 3 & $\begin{array}{l}\text { Wilson et al. (1981); } \\
\text { Jackson et al. (1991) }\end{array}$ \\
\hline $\begin{array}{l}\text { Number of shaded } \\
\text { epitopes per bound } \\
\text { IgG }\end{array}$ & $e^{*}$ & 3 & $\begin{array}{l}\text { Bernoulli }(0.5) \text { with } \\
e^{*} \in\{3,6\}\end{array}$ & $\begin{array}{l}\text { Otterstrom et al. } \\
(2014)\end{array}$ \\
\hline $\begin{array}{l}\text { Number of SA } \\
\text { receptors per RBC }\end{array}$ & $b$ & $4.5 \cdot 10^{5}$ & $\begin{array}{l}\text { Discrete } \\
\text { Unif }\left(4 \cdot 10^{5}, 5 \cdot 10^{5}\right)\end{array}$ & $\begin{array}{l}\text { Aich et al. (2011); } \\
\text { Aoki (2017) }\end{array}$ \\
\hline $\begin{array}{l}\text { Number of shaded } \\
\text { SA receptors per } \\
\text { bound virus }\end{array}$ & $b^{*}$ & 34 & Sampled from model & See Methods \\
\hline $\begin{array}{l}\text { SA-HA association } \\
\text { rate constant }\end{array}$ & $k_{\mathrm{ass}}^{\mathrm{RBC}}$ & $2 \cdot 10^{-6} \mathrm{~s}^{-1}$ & $\begin{array}{l}\mathcal{N}\left(2 \cdot 10^{-6},(0.4\right. \\
\left.\left.10^{-6}\right)^{2}\right) \mathrm{s}^{-1}\end{array}$ & $\begin{array}{l}\text { Takemoto et al. } \\
(1996)\end{array}$ \\
\hline $\begin{array}{l}\text { SA-HA dissociation } \\
\text { rate constant }\end{array}$ & $k_{\text {diss }}^{\mathrm{RBC}}$ & $\begin{array}{l}2 \cdot \\
10^{-4} \mathrm{nM}^{-1} \mathrm{~s}^{-1}\end{array}$ & $\begin{array}{l}\mathcal{N}\left(2 \cdot 10^{-4},(0.4\right. \\
\left.\left.10^{-4}\right)^{2}\right) \mathrm{nM}^{-1} \mathrm{~s}^{-1}\end{array}$ & $\begin{array}{l}\text { Takemoto et al. } \\
(1996)\end{array}$ \\
\hline $\begin{array}{l}\text { RBC agglutination } \\
\text { rate constant }\end{array}$ & $k_{\text {agg }}$ & $2 \cdot 10^{6} \mathrm{~s}^{-1}$ & $\operatorname{Unif}\left(0.4 \cdot 10^{6}, 13 \cdot 10^{6}\right) \mathrm{s}^{-1}$ & Estimated from data \\
\hline
\end{tabular}


a

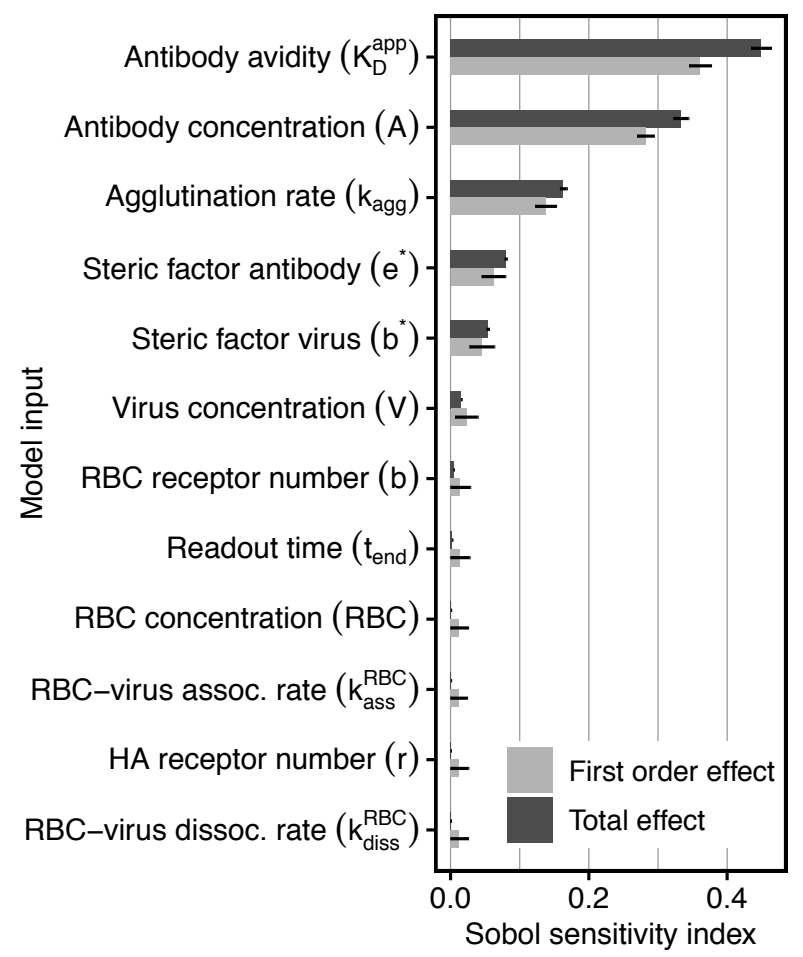

b

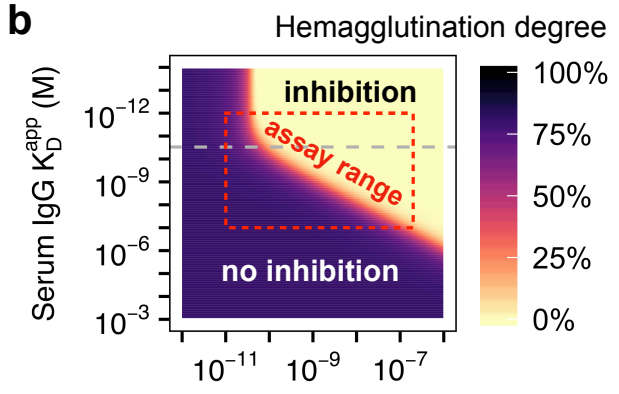

Diluted serum IgG conc. (M)

C

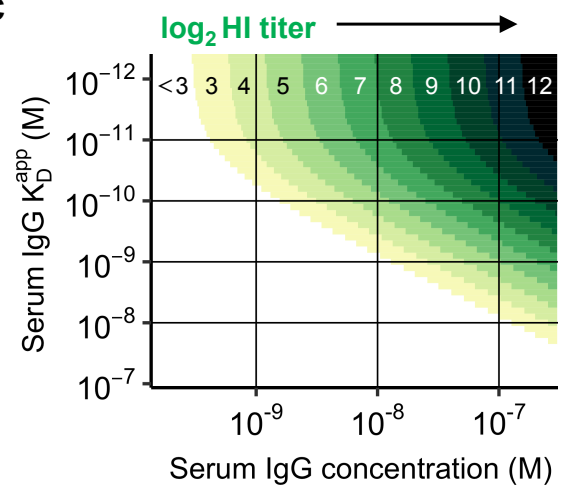

Figure 2. Model sensitivity and resolution of the hemagglutination inhibition assay for influenza H1N1pdm09. (a) Sensitivity analysis using Sobol indices. First-order effects show only the linear contribution to the total variance in hemagglutination degree (they sum up to 1), whereas total effects consider also interactions (see Methods for details). (b) Predicted degree of hemagglutination for different IgG concentrations and apparent dissociation constants $K_{\mathrm{D}}^{\text {app }}$. The red box indicates the usual assay range, bounded by the biological range of $K_{\mathrm{D}}^{\mathrm{app}}=0.001-100 \mathrm{nM}$, and the gray dashed line indicates $K_{\mathrm{D}}^{\mathrm{app}}=0.03 \mathrm{nM}$. (c) Predicted HI titers for the biological range of influenza-specific serum IgG and $K_{\mathrm{D}}^{\mathrm{app}}$. Colored areas correspond to titers shown on top.

$2015)$ and $K_{\mathrm{D}}^{\mathrm{app}}=0.001-300 \mathrm{nM}$ (Lee et al., 2016). For experimental conditions, we aimed to generously cover experimental variability $(0.375-1.5 \%(\mathrm{v} / \mathrm{v}) \mathrm{RBC}$ suspension, $3-7$ HA units influenza virus and a readout time of 30 to $45 \mathrm{~min}$ ). For model parameters, we considered measurement uncertainty and biological variability as described in the literature.

Sensitivity analysis showed that avidity and serum $\operatorname{IgG}$ concentration are the most influential factors for the model's output (Figure 2a). Variability in RBC and virus concentration, as well as in readout time, contribute very little to the total variance. The model is also robust to uncertainty in all model parameters except for the kinetic agglutination rate of RBCs, which we varied within the $95 \%$ highest probability density interval estimated from our calibration data (see Methods). Other relevant factors were the ability of IgG to bind two HA receptors simultaneously and the number of RBC receptors that are covered by one bound virion. Hence, the model's predictions are dominated by the measured input quantities, despite uncertainties on mechanisms and parameters.

According to the degree of hemagglutination, the model predicts a clear separation between 
inhibition and no inhibition - partial inhibition occurs only within a small range of IgG concentration and avidity (Figure 2b). Thus, the well-known binary nature of the assay is captured. Using an initial virus concentration of 4 HA units as defined by WHO ensures both high sensitivity and robustness, whereas 8 HA units or more increase robustness but lower sensitivity (Supplementary Figure S1e). In addition, the model predicts a yet unknown property of the $\mathrm{HI}$ assay within its range. For avidities $K_{\mathrm{D}}^{\mathrm{app}} \geq 0.03 \mathrm{nM}$, hemagglutination quantifies a combination of IgG concentration and avidity, but for very high avidities $K_{\mathrm{D}}^{\text {app }}<0.03 \mathrm{nM}$, the assay only detects changes in IgG concentration (Figure $\mathbf{2 b}$ ).

Correspondingly, within the linear range for $K_{\mathrm{D}}^{\mathrm{app}} \geq 0.03 \mathrm{nM}$, a doubling in IgG concentration or avidity results in a doubling of the predicted HI titer (Figure 2c). In other words, a two-times lower antibody avidity can be compensated by a two-times higher antibody concentration. However, this only applies to the linear range. The exact relationship depends on the considered avidity and concentration ranges (Figure 2c). For instance, in our experimental setup, where serum samples were 1:8 prediluted and then two-fold serially diluted, the minimal concentration to reach seroprotection (HI titer $\geq 40$ ) with an IgG avidity of $K_{\mathrm{D}}^{\text {app }}=1 \mathrm{nM}$ is an IgG serum concentration of $104 \mathrm{nM}(16 \mu \mathrm{g} / \mathrm{mL})$, whereas for $K_{\mathrm{D}}^{\text {app }}=10 \mathrm{nM}$, the minimal seroprotective IgG concentration increases to $1018 \mathrm{nM}(153 \mu \mathrm{g} / \mathrm{mL})$. These values correspond to approximately $0.1-10 \%$ of total serum IgG (Gonzalez-Quintela et al., 2008) and lie within the observed range for influenza-specific serum IgG concentrations in seroprotected healthy adults (Eidem et al., 2015). The model also suggests why HI titers above $8192\left(=13 \mathrm{in} \log _{2}\right)$ are rarely observed. Even for a high serum IgG concentration of $1000 \mathrm{nM}(150 \mu \mathrm{g} / \mathrm{mL})$, such high titers require antibody avidities in the $\mathrm{fM}$ range, but influenza-specific monoclonal antibody affinities in vaccinated healthy adults lie in the nM range (Lee et al., 2016). Therefore, we conclude that the model yields robust predictions in the applicable assay range, and reveals new quantitative aspects of the HI assay.

\section{Inference of neutralizing antibody avidities in HSCT patients}

Next, we applied our model to infer avidities from ELISA-detected serum IgG concentrations and HI titers in HSCT patients (patient characteristics are summarized in Table 2). Specifically, we used a Bayesian approach that accounts for uncertainties due to ELISA measurement error and discretization in HI titers (see Methods for details). Model parameters were fixed for all serum samples (Table 1), assuming that differences in HI titer arise mostly from differences in serum IgG and avidity as suggested by our sensitivity analysis (Figure 2a).

Measurements were available from 45 patients at five time points before (d0) and after (d7, d30, d60, d180) the first vaccination with 221 serum samples in total. Patients received two doses of non-adjuvanted trivalent seasonal influenza vaccine on d0 and d30 (see Methods). HI titers and IgG concentrations were significantly correlated (Kendall's $\tau=0.69, p<10^{-15}$, rank correlation for ordinal data; Figure 3a). However, especially moderate HI titer values of 32 
and $64\left(\log _{2} \mathrm{HI}\right.$ titer 5 and 6) showed a large spread in serum IgG, indicating variable antibody avidities.

For serum samples with HI titers below assay resolution (HI titer $<8$ ), we could only infer an upper bound for the avidity (it could be lower, but not higher). This affected 23 serum samples from seven patients. Analogously, for serum samples with $K_{\mathrm{D}}^{\mathrm{app}} \leq 0.03 \mathrm{nM}$, we could, in principle, only report a lower bound, but all inferred avidities for our patient cohort exceeded this threshold. In 24 samples, inferred $K_{\mathrm{D}}^{\text {app }}$ values showed very large uncertainty (approximately $\pm 100 \%$ ) due to large measurement error in ELISA measurements; we excluded these samples from further analysis. In the remaining samples, the average uncertainty in $K_{\mathrm{D}}^{\text {app }}$ values was $\pm 27 \%$ (interquartile range, IQR $25-28 \%$ ). This mostly results from discretized HI titers because the average uncertainty in ELISA measurements was only $\pm 10 \%$, as normally observed for ELISA. In addition, posterior distributions of inferred avidities were log-normally distributed (Supplementary Figure S2), indicating that we computed meaningful credibility intervals by sampling (see Methods).

In summary, we were able to reliably infer 197 avidities from in total 43 patients (89\% of analyzed samples). Inferred avidities ranged from $K_{\mathrm{D}}^{\text {app }}=0.08 \mathrm{nM}$ to $\geq 37 \mathrm{nM}$ (upper bound), with a median of $1.7 \mathrm{nM}$ and IQR $0.9-2.5 \mathrm{nM}$, thus covering almost the full biological range (see Figure 2c). Inferred avidities and HI titers were significantly correlated (Kendall's $\tau=0.56$, $P<10^{-15}$ ), although the correlation was weaker than for $\operatorname{IgG}$ concentrations (Figure 3a).

\section{Inferred avidities correlate with experimentally determined avidities}

To validate our model, we measured serum IgG avidities specific to H1N1pmd09 in 59 serum samples from a total of 12 HSCT patients. We performed ELISA-based elution assays that quantify the fraction of IgG remaining bound after $3 \mathrm{~h}$ incubation with $4 \mathrm{M}$ urea, yielding a measure for the overall binding strength of serum IgG to H1N1pmd09 in the form of an index between 0 (low avidity) and 1 (high avidity). The inferred apparent dissociation constants $K_{\mathrm{D}}^{\text {app }}$ and the experimentally determined avidity indices were significantly correlated (Pearson $\rho=$ $0.54, p<10^{-4}$, Figure 3b). We detected one outlier patient (standardized residuals $\approx 3$ ) whose serum did not show HI activity at any time point (Figure 3b), suggesting that the ELISA assay detected non-neutralizing $\operatorname{IgG}$ in this patient.

Next, we asked if the inferred avidities could help explain patient-specific responses to vaccination over time. Given the uncertainty in inferred $K_{\mathrm{D}}^{\mathrm{app}}$-values due to discretized HI titer observations, we could detect fold changes in avidity of approximately $>1.5$ or $<0.5$ (except for samples below the HI assay's resolution with titer $<8$ ). Experimental and inferred avidities indeed distinguished different types of patient responses, for example, where both IgG concentration and avidity increased after vaccination (patient 1 in Figure 3c) or where an increase in $\mathrm{HI}$ titer was mostly explained by an increase in $\mathrm{IgG}$ concentration (patient 2). Moreover, we 
a Inferred $\mathrm{K}_{\mathrm{D}}^{\mathrm{app}}(\mathrm{nM})$
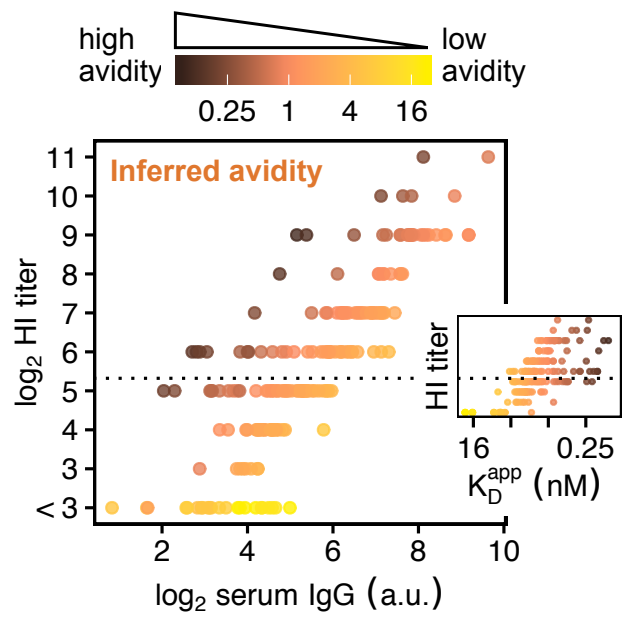

b

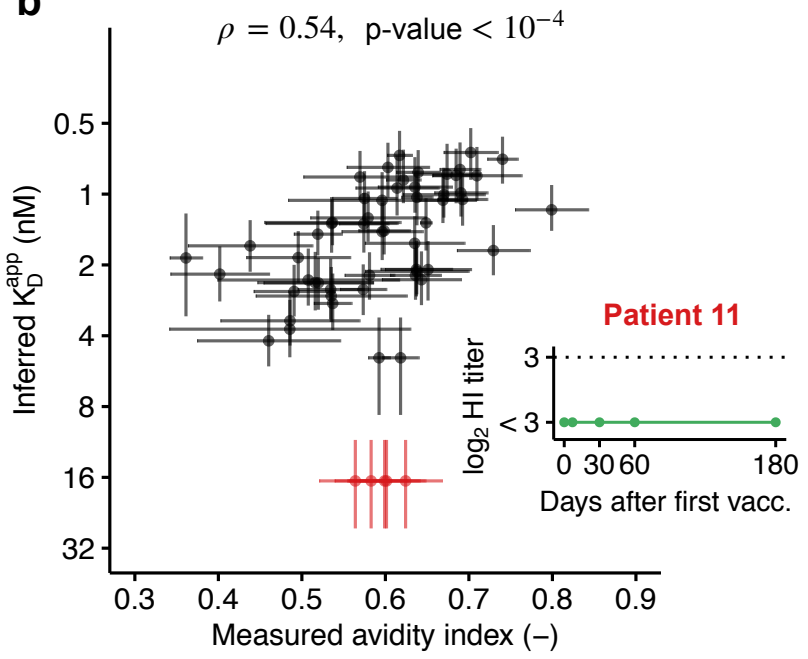

C

Measured avidity

Inferred avidity

Serum IgG conc.

HI titer

Patient 1
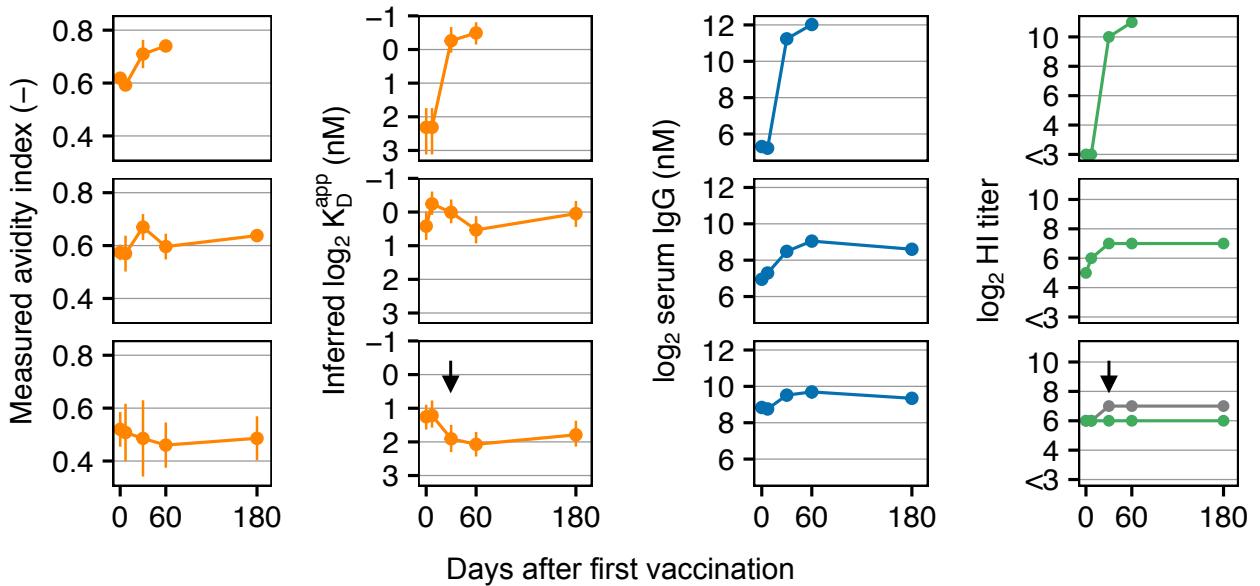

Days after first vaccination

Figure 3. Inference of antibody avidities in HSCT patients. (a) ELISA-detected anti-H1N1pmd09 serum IgG concentration, HI titers and corresponding inferred apparent dissociation constants $K_{\mathrm{D}}^{\mathrm{app}}$ from 197 serum samples from 43 HSCT patients. The dashed line indicates the seroprotection threshold (HI titer $\geq 40$ ). (b) Correlation of inferred and experimentally determined avidities in 59 serum samples from 12 HSCT patients (Pearson $\rho=0.54,95 \% \mathrm{CI}=[0.31,0.70]$ ). Data show mean and standard deviation for avidity indices from two experiments (each performed in duplicates) and the maximum a posteriori (MAP) estimates and uncertainty range due to discretized HI titer measurements and ELISA measurement error for inferred dissociation constants $K_{\mathrm{D}}^{\text {app }}$ (see Methods for details on inference). Avidity indices correspond to the fraction of $\mathrm{H} 1 \mathrm{~N} 1 \mathrm{pmd} 09$-specific serum IgG remaining bound after $4 \mathrm{M}$ urea treatment. Patient 11 was identified as an outlier, probably because ELISA detected non-neutralizing IgG; the patient showed no HI activity at any time point. (c) Example patients with different types of responses to vaccination. In patient 3, we detected an increase in non-neutralizing IgG on d30. The predicted HI titer for the observed increase in IgG (shown in grey) is twice as high as the actually observed titer (green). For all 12 patients with experimentally determined avidities see Supplementary Figure S3. 
identified one patient that produced non-neutralizing $\operatorname{IgG}$ upon vaccination (patient 3 in Figure 3c): In this patient, the ELISA detected an increase in IgG concentration that leads to HI titer doubling according to our model predictions. However, the HI titer did not increase at any time point (Figure 3c), suggesting that the ELISA-detected IgGs had no HI activity (were nonneutralizing). The inferred apparent dissociation constant $K_{\mathrm{D}}^{\text {app }}$ refers to neutralizing IgG-virus interactions only, and its value is biased towards lower avidity if the measured IgG concentration also includes non-neutralizing IgGs. Nevertheless - in contrast to patient 11 who did not show HI activity at any time point (Figure 3b) - the inferred avidities for patient 3 approximately mirrored the experimentally determined avidity indices, probably because this patient showed high HI titer and serum IgG levels before vaccination and only a small increase in non-neutralizing IgGs after vaccination, indicating that previously acquired neutralizing IgGs dominated the avidity measurements (Figure 3c). Thus, apparent serum avidities inferred by our model-based approach were in good accordance with experimentally determined avidity indices. However, as one would expect, if non-neutralizing IgG dominates in serum, the results are not directly comparable because the inferred avidity refers to neutralizing $\operatorname{IgG}$ with $\mathrm{HI}$ activity.

\section{Detection of vaccine-induced affinity maturation in HSCT patients}

Next, we compared the vaccine-induced increase in inferred avidities in all investigated HSCT patients and identified candidates for successful GC formation and affinity maturation (Figure 4). Since the establishment of GCs takes approximately seven days (De Silva and Klein, 2015), we considered an increase in IgG concentration and avidity on d30 or d60 as indicative for GC formation (patients were vaccinated on $\mathrm{d} 0$ and $\mathrm{d} 30$; see Methods).

The response characteristics of the patient cohort are shown in Figure 4a. Taking uncertainty in inferred $K_{\mathrm{D}}^{\text {app }}$ values into account, eight patients showed a detectable increase in avidity on d30 and/or d60, of which only one showed no increase in serum IgG (Figure 4b). This suggests that vaccination induced GC formation and affinity maturation in seven patients (including patient 1 in Figure 3c), although serum avidity returned back to baseline on d180 in most of the patients, suggesting that vaccination failed to induce a sustained production of high-avidity antibodies by long-lived plasma cells. In two out of seven patients, avidity increased already on d7, suggesting that reactivated memory B cells released high-avidity IgG. Over all patients showing a detectable increase in avidity at any time point after vaccination $(n=11)$, we observed a timedependent increase with the largest increase on d60, i.e. after the booster dose (Figure 4c). Of these patients, only one showed an increase in avidity on $\mathrm{d} 7$ but not at later time points and two patients only on d180. In conclusion, most patients showed affinity maturation dynamics consistent with our understanding of GC dynamics (De Silva and Klein, 2015).

We could not detect a significant increase in avidity in 24 patients, although 15/24 patients showed an increase in serum $\operatorname{IgG}$ on $\mathrm{d} 30$ or $\mathrm{d} 60$ (such as patient 2 in Figure 3c). We identified 
a

$\begin{array}{rccccc}\text { Time } & \text { d0 } & \text { d7 } & \text { d30 } & \text { d60 } & \text { d180 } \\ \text { Total } & 45 & 42 & 45 & 45 & 45 \\ \text { Seroprotected } & 20(44 \%) & 23(55 \%) & 27(60 \%) & 28(62 \%) & 26(58 \%) \\ \text { Seroconverted } & - & 5(12 \%) & 12(27 \%) & 13(29 \%) & 5(11 \%)\end{array}$

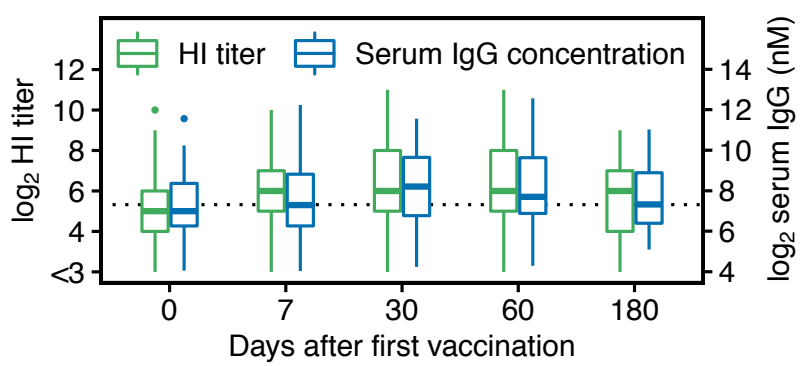

C

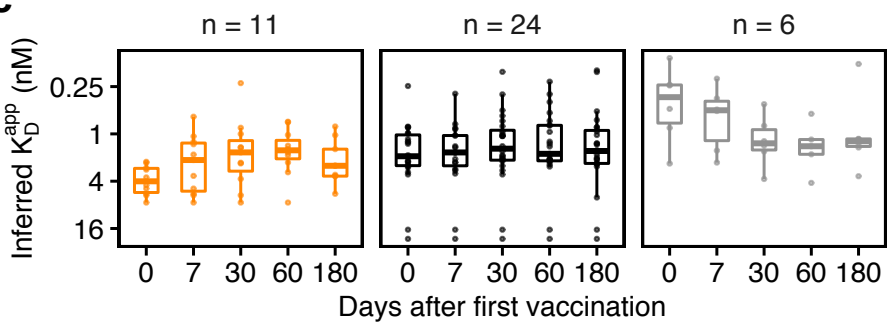

b

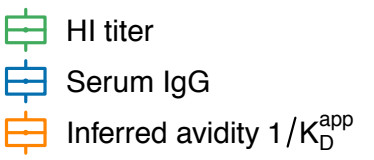

d

Inferred avidity $1 / K_{D}^{\text {app }} \quad$ Serum IgG
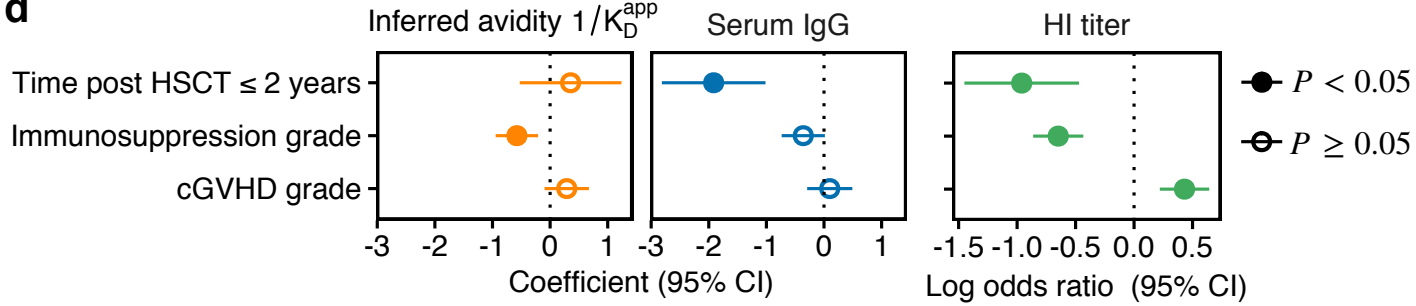

Figure 4. Vaccine response to influenza H1N1pmd09 in HSCT patients. (a) Patients were vaccinated on $\mathrm{d} 0$ and $\mathrm{d} 30$ with a non-adjuvanted trivalent influenza vaccine. Serum IgG was measured by ELISA with whole-virus coating (see Methods). (b) Fold changes in HI titer, serum IgG and avidity $\left(1 / K_{\mathrm{D}}^{\mathrm{app}}\right)$ in all patients with a detectable increase in inferred avidity on d30 and/or d60. (c) Comparison of inferred avidities between patients with a detectable increase in avidity at any time point after vaccination (left), patients with no detectable increase (middle), and patients with a detectable increase in non-neutralizing IgG (right). (d) Estimated effects of criteria for compromised immune response according to the CDC. Effects were estimated from multiple regression on log2-transformed values controlling for sex and age (model for continuous data for serum IgG and avidity, model for ordinal data for HI titers, see Methods). Time after HSCT was encoded as a binary variable ( 1 for HSCT $\leq 2$ years and 0 for HSCT $>2$ years). Immunosuppression grade and cGVHD grade ranging from 0 (no immunosuppression/cGVHD) to 3 (severe immunosuppression/cGVHD) were encoded as ordered categorical variables with grade 0 as reference. P-values $<0.05$ were considered significant. 
six patients as non-neutralizing IgG producers, including patient 3 in Figure 3c. These patients showed no increase in $\mathrm{HI}$ titer despite showing an increase in IgG concentration that resulted in a significant bias towards lower avidities (Supplementary Figure S4a). We excluded 4/45 patients as they showed too large measurement uncertainty in ELISA-detected IgG concentrations on several time points (see above).

In summary, although 30 patients showed an increase in serum IgG on d30 and/or d60, only $7 / 30$ patients $(23 \%)$ are candidates for vaccine-induced affinity maturation, and $6 / 30$ patients (20\%) showed vaccine-induced production of non-neutralizing IgG.

\section{Association with criteria for compromised immune response}

Finally, we investigated differences in inferred avidity, serum IgG concentration, and HI titer between different patient groups. We considered criteria for compromised immune response as defined by CDC, which are known to be associated with immune cell proliferation, affinity maturation and antibody production (Brunette et al., 2015; Ogonek et al., 2016): (i) first two years post transplantation (time post $\mathrm{HSCT} \leq 2$ years), (ii) intake of immunosuppressive drugs quantified by immunosuppression grade ranging from 0 (no immunosuppression) to 3 (severe immunosuppression), and (iii) chronic graft-versus-host disease (cGVHD) quantified by cGVHD grade between 0 (no cGVHD) and 3 (severe cGVHD) according to NIH criteria (Filipovich et al., 2005). We investigated effects on baseline (i.e. levels before vaccination) and response (relative increase from baseline) in a multivariable linear regression analysis with the three criteria as explanatory variables and additionally controlling for sex and age. Regression was performed on $\log 2$-transformed values using models for continuous data for serum $\operatorname{IgG}$ and avidity, whereas HI titers were treated as ordinal data (see Methods). When analyzing the vaccine-induced increase in avidity, we excluded non-neutralizing IgG responders $(n=6$, Figure $4 \mathbf{c}$ ) because their biased inferred avidities are not indicative of affinity maturation.

Early transplant patients (time post $\mathrm{HSCT} \leq 2$ years) showed significantly lower serum $\operatorname{IgG}$ $\left(-1.92 \pm 0.46, P=1.8 \cdot 10^{-4}\right)$ and HI titer baseline levels (log odds ratio $-0.96 \pm 0.25$, $P=1.2 \cdot 10^{-4}$ ) than patients with HSCT $>2$ years (Figure 4d). At the time of this study, the annual influenza vaccine contained H1N1pmd09 already for five years. Therefore, it is likely that patients with HSCT $>2$ years acquired durable H1N1pmd09-neutralizing antibodies in previous seasons. Yet, early transplant patients did not show significantly different baseline avidities compared to patients with HSCT $>2$ years $(0.36 \pm 0.45, P=0.43)$, potentially because previous vaccinations did not induce long-term production of high-avidity antibodies. This is consistent with some patient observations in the present season (Figure $\mathbf{4 b}$ ).

Most patients in our cohort under immunosuppression received cell division inhibitors such as prednisone or mycophenolate mofetil (MMF) and calcineurin inhibitors such as tacrolimus or cyclosporine A that lower T cell activity, while few patients received rituximab, an anti-CD20 antibody that leads to B-cell depletion (Table 2). Immunosuppression grade was associated 
with lower serum $\operatorname{IgG}$ baseline levels, although not significantly $(-0.36 \pm 0.19, P=0.07)$, and with significantly lower baseline avidities $\left(-0.58 \pm 0.19, P=4.0 \cdot 10^{-3}\right)$ and baseline titers ( $\log$ odds ratio $-0.65 \pm 0.11, P=3.1 \cdot 10^{-9}$, Figure 4d). Since we did not know the patients' medical history, we could not investigate whether the low baseline levels are explained by immunosuppressive drug treatment or an underlying disease during a former encounter with H1N1pmd09. For instance, patients might have suffered from GVHD in previous seasons, which required treatment with immunosuppressive drugs. In the current season, we could not detect any effects on the vaccine-induced increase in serum IgG or avidity, probably due to lack of power. We also did not detect significant effects of cGVHD on avidity or serum IgG, but we found a positive effect on $\mathrm{HI}$ baseline titers ( $\log$ odds ratio $0.43 \pm 0.11, P=6.2 \cdot 10^{-5}$ ).

In conclusion, the time after transplantation showed a significant, positive effect on baseline levels in serum IgG and HI titers but no significant effect on baseline avidity. Instead, patients under immunosuppression showed significantly lower baseline avidities with an estimated effect size of approximately $-0.6 \pm 0.2$ per immunosuppression grade (for log2-transformed $1 / K_{\mathrm{D}}^{\text {app }}$ values). This means, that in our HSCT patient population, patients with immunosuppression grade 2 showed approximately two-fold lower baseline avidities than patients without immunosuppression, while patients with immunosuppression grade 3 show three- to four-fold lower baseline avidities (Supplementary Figure S5). We did not detect significant differences in the vaccine-induced increase in serum IgG or avidity, potentially because the number of responders in the investigated HSCT population was too low (only 13 patients showed seroconversion).

\section{Discussion}

The HI assay is a well-established gold standard method, and yet, little is known on the relationship between HI titer, serum antibody concentration, and avidity. Mathematical models of cell agglutination by antibody cross-linking have been previously reported (Ming et al., 1965; Dolgosheina et al., 1992) and applied to guide the design of immunoassays (Kylilis et al., 2019). Here, we present an extension to a system of three components consisting of antibodies, viruses, and cells. Our model captures known properties of the HI assay and provides a biophysical explanation for why the HI assay has become the gold standard in serological studies. First, the assay is equally sensitive to both antibody concentration and avidity. Only for extremely high avidities $\left(K_{\mathrm{D}}^{\text {app }} \leq 0.03 \mathrm{nM}\right.$ ), it detects only changes in concentrations. Second, the assay is robust to pipetting errors or other experimental variabilities in $\mathrm{RBC}$ and virus concentration.

Model development required simplifying assumptions that necessarily limit the model's applicability. For example, we neglected IgM antibodies because IgMs show lower serum concentration than IgGs (Gonzalez-Quintela et al., 2008). When IgM concentration is high while IgG concentration is low, for example, on $\mathrm{d} 7$ after vaccination in a naive subject, modeling the contribution of IgM to the HI titer may be necessary. However, the model can describe mono- and 
bivalent IgG binding, and it can distinguish bivalent binding to the same HA receptor from bivalent binding to two different HA receptors, resulting in lower $K_{\mathrm{D}}^{\text {app }}$ compared to monovalent binding (Edwards and Dimmock, 2000; Williams et al., 2018). Note also that we assume that the simultaneous binding of HA receptors on two different virus particles is rare under $\mathrm{HI}$ assay conditions and thus negligible.

Regarding our experimental assays, we note that most influenza-specific IgG antibodies bind to the immunodominant HA globular domain (part of HA1) that harbors the SA binding site and attaches the virus to host cells (Wilson et al., 1981; Gerhard et al., 1981). Although we used whole-virus for ELISA coating, we observed a similar correlation between ELISA-detected serum IgG and HI titers as previous studies for HA and HA1 coating (Li et al., 2014; Trombetta et al., 2018). This is in line with studies on immunodominance showing that antibody response to influenza is dominated by HA-specific neutralizing $\mathrm{IgG}$, and the HA globular domain is favored over the HA stalk domain, neuraminidase or other proteins on influenza (Altman et al., 2015; Angeletti et al., 2017). However, detection of both neutralizing and non-neutralizing IgG can bias our results towards lower avidities; this possibility could be evaluated with surface plasmon resonance or calorimetry measurements.

The model allows the inference of serum avidities from ELISA-detected IgG concentrations and HI titers, which are simpler, faster, and cheaper to measure than antibody avidities, especially in larger populations. In our experimental setup where HI titers were determined in two-fold serial dilutions, we were able to estimate neutralizing serum avidities with a precision of approximately $\pm 25-30 \%$. A limitation of our approach is that we cannot distinguish whether $\mathrm{HI}$ titers below assay resolution (HI titer $<8$ ) correspond to non-neutralizing $\mathrm{IgG}$ (which could potentially have high avidity but not to HA) or to neutralizing IgG below assay resolution (with low avidity or low concentration).

We inferred serum avidities for our HSCT patient cohort because serum avidities serve as markers for GC formation and affinity maturation in vaccine studies in general (Khurana et al., 2011, 2012; Eidem et al., 2015; Khurana et al., 2019). For example, it has been shown that in response to influenza A, the average HA-specific affinity of GC B cells is correlated with the HA-specific apparent avidity of serum antibodies (Frank et al., 2015) and that avidity of serum antibodies is important for protection (Olszewska et al., 2000; Polack et al., 2003). However, it is unknown to which extent and with which dynamics influenza vaccination against H1N1pmd09 induces affinity maturation in HSCT patients. Previous studies in healthy adults vaccinated against H1N1pmd09 showed that serum avidity to HA1 peaks at $21-28 \mathrm{~d}$ after vaccination and decreases almost back to baseline on d180 (Eidem et al., 2015; Khurana et al., 2019). We observed a similar behavior among those HSCT patients that showed a detectable increase in avidity, although, in contrast to healthy subjects, HSCT patients received a booster dose on $\mathrm{d} 30$. Over all patients, we observed the largest increase in avidity on d60, suggesting that the booster dose might be important for vaccine-induced affinity maturation. 
Only a few patients showed a vaccine-induced increase in avidity. This may be because the number of responders was low or because the increase was below our detection limit (fold change $<1.5$ ). Whether poor vaccine-induced affinity maturation is responsible for the poor effectiveness of seasonal influenza vaccination is currently under debate (Ellebedy, 2018; Arevalo et al., 2020; Hoehn et al., 2021). However, we observed consistent effects: among 32 (13) patients with a vaccine-induced increase in serum IgG on d30 or d60 (seroconversion on d60), we identified only seven (five) candidates for vaccine-induced affinity maturation. Thus, vaccineinduced increases in $\mathrm{HI}$ titer were mostly explained by increased IgG concentrations. These results might not apply to other populations, especially because hampered affinity maturation is likely in HSCT patients (Ogonek et al., 2016). Yet, the correlation between HI titers and serum IgG in healthy adults is only slightly lower than in our population (reported $95 \%$ confidence intervals for Pearson's $\rho=[0.55,0.67]$ for ELISA-measured IgG with H1N1pmd09-specific HA coating compared to Pearson's $\rho=[0.77,0.87]$ for our results) (Trombetta et al., 2018), suggesting that also in healthy populations differences in HI titers are mostly explained by differences in serum IgG concentration.

Interestingly, all patients identified as non-neutralizing $\operatorname{IgG}$ producers showed relatively high $\mathrm{HI}$ titers (ranging from $32-1024$ with a geometric mean titer of 128) and high neutralizing avidities before vaccination (Figure 4c). Even if we detected both neutralizing and non-neutralizing IgG on $\mathrm{d} 0$ in these patients, this would bias the inferred $K_{\mathrm{D}}^{\text {app }}$ values towards lower avidities, which means that the actual neutralizing baseline avidities could be even higher. This observation supports computer simulations suggesting that preexisting antibodies that mask immunodominant epitopes lead to the production of antibodies specific to less accessible epitopes (Meyer-Hermann, 2019). This might be particularly important for the generation of so called broadly neutralizing antibodies that target the membrane proximal HA stalk domain, which is less accessible but more conserved than the HA globular domain (Krammer, 2019). These antibodies show high potential in vivo despite poor neutralization activity in vitro (Krammer, 2016). Therefore, additional studies might investigate whether preexisting antibodies with high neutralizing avidities against the HA globular domain lead to the vaccine-induced production of antibodies against the HA stalk domain.

The precision of our inference approach was also sufficient to detect differences in baseline avidities between HSCT patients with and without immunosuppressive treatment. Excluding the two patients without $\mathrm{HI}$ activity at any time point from our analysis only slightly affects the detected association between immunosuppression grade and avidity $(-0.39 \pm 0.16, p=0.02)$. Previous studies in healthy adults showed that repeated annual vaccination against H1N1pmd09 can increase H1-specific baseline avidities (Eidem et al., 2015; Khurana et al., 2019). Patients with ongoing immunosuppression or CGVHD are recommended to receive yearly influenza vaccination (Hilgendorf et al., 2011) but in this study, we could not investigate further whether differences in baseline avidities were associated with immunosuppressive treatment at the time of vaccination, or simply with repeated annual vaccinations against H1N1pmd09. 
Interestingly, HI titers were negatively associated with immunosuppression grade but positively with cGVHD grade. Patients with cGVHD show disturbed B cell homeostasis, persistent B cell activation, and elevated levels of B-cell activating factor (BAFF), which promotes survival and differentiation of activated B cells (Sarantopoulos et al., 2007, 2009; Greinix et al., 2008; Jacobson et al., 2014). However, several studies reported no significant effect of cGVHD on vaccine response to H1N1pmd09 (Issa et al., 2011; Engelhard et al., 2011; Gueller et al., 2011) or a negative effect (Roll et al., 2012; Mohty et al., 2011). Information on the patients' vaccination history, medication and disease state at the time of previous vaccinations would be required to better understand this association.

Overall, we argue that the new analysis capabilities afforded by our biophysical model for the $\mathrm{HI}$ assay not only generate detailed insights and hypotheses on vaccination responses in small patient cohorts as here. Because the model requires only easy-to-establish measurements as inputs, we anticipate that it can also refine the analysis in larger vaccine studies.

\section{Methods}

\section{Model derivation}

\section{Assay step 1: binding of antibodies to virus}

We model the formation of antibody-epitope complexes, denoted by $C$, as a diffusion-controlled reaction between viruses and antibodies, following the model of antibody-virus interaction proposed by Groth (1963). For complex formation, free antibodies, $A$, need to successfully collide with free influenza virus particles, $V$. In addition, antibody-epitope complex formation depends on the probability of epitopes being unbound, denoted by $\phi$. The dynamics of complex formation is thus given by:

$$
\frac{d C(t)}{d t}=k_{\mathrm{ass}} \cdot A(t) \cdot V(t) \cdot \phi(t)-k_{\mathrm{diss}} \cdot C(t),
$$

where $k_{\text {ass }}$ and $k_{\text {diss }}$ are kinetic rate constants for association and dissociation, respectively. Note that some IgG antibodies bind bivalently to hemagglutinin, resulting in higher antibody affinities compared to their monovalent Fab fragments due to lower macroscopic dissociation rates (Edwards and Dimmock, 2000; Williams et al., 2018). This antibody valency is lumped into the macroscopic dissociation constant $k_{\text {diss }}$.

The total number of epitopes is proportional to the total virus concentration $V_{\text {tot }_{1}}$ (where '1' indicates the first step of the assay), the average number of hemagglutinin receptors per virus, $r$, and the number of identical binding sites per hemagglutinin, $e(e=3$ since hemagglutinin is a 
homotrimer). With $e^{*}$ being the number of epitopes bound or shaded by one antibody molecule, the fraction of unbound epitopes is:

$$
\phi(t)=\frac{e \cdot r \cdot V_{\mathrm{tot}_{1}}-e^{*} \cdot C(t)}{e \cdot r \cdot V_{\mathrm{tot}_{1}}} .
$$

1 We assume that cross-linking of virus particles by antibodies is rare for the considered concentrations, such that the concentration of virus particles $V$ remains approximately the same during the experiment, i.e., $V \approx V_{\mathrm{tot}_{1}}$. In addition, the mass balance for antibodies is $A_{\mathrm{tot}_{1}}=$ $A(t)+C(t)$. Substituting into the dynamics of complex formation leads to:

$$
\frac{d C(t)}{d t}=\frac{k_{\mathrm{ass}}}{e \cdot r} \cdot\left[A_{\mathrm{tot}_{1}}-C(t)\right] \cdot\left[e \cdot r \cdot V_{\mathrm{tot}_{1}}-e^{*} \cdot C(t)\right]-k_{\mathrm{diss}} \cdot C(t) .
$$

Since the average number of epitopes per virus particle $e \cdot r$ is constant, the dynamics is equivalent to a reversible bimolecular reaction following mass action kinetics with apparent dissociation constant $K_{\mathrm{D}}^{\text {app }}=e \cdot r \cdot \frac{k_{\text {diss }}}{k_{\text {ass }}}$. We assume that antibody-virus binding is fast, such that after the incubation time the system is at steady-state. At steady-state, the complex concentration $C^{\text {eq }}$ fulfills

$$
0=\left[A_{\mathrm{tot}_{1}}-C^{\mathrm{eq}}\right] \cdot\left[e \cdot r \cdot V_{\mathrm{tot}_{1}}-e^{*} \cdot C^{\mathrm{eq}}\right]-K_{\mathrm{D}}^{\mathrm{app}} \cdot C^{\mathrm{eq}} .
$$

We exploit the analytic solution to this quadratic equation in $C^{\text {eq }}$ to compute the fraction of covered hemagglutinin epitopes at equilibrium, $\theta$, defined as:

$$
\theta=\frac{e^{*} \cdot C^{\mathrm{eq}}}{e \cdot r \cdot V_{\mathrm{tot}_{1}}}
$$

to obtain:

$$
\begin{aligned}
\theta= & \frac{e r V_{\mathrm{tot}_{1}}+e^{*} A_{\mathrm{tot}_{1}}+K_{\mathrm{D}}^{\mathrm{app}}}{2 e r V_{\mathrm{tot}_{1}}}- \\
& \frac{\sqrt{e^{2} r^{2} V_{\mathrm{tot}_{1}}^{2}+\left(K_{\mathrm{D}}^{\mathrm{app}}\right)^{2}+e^{* 2} A_{\mathrm{tot}_{1}}^{2}-2 e r V_{\mathrm{tot}_{1}} e^{*} A_{\mathrm{tot}_{1}}+2 K_{\mathrm{D}}^{\mathrm{app}} e r V_{\mathrm{tot}_{1}}+2 K_{\mathrm{D}}^{\mathrm{app}} e^{*} A_{\mathrm{tot}_{1}}}}{2 e r V_{\mathrm{tot}_{1}}} .
\end{aligned}
$$

\section{Assay step 2: hemagglutination}

When RBC suspension is added to the system, two processes happen simultaneously: viruses bind to SA-linked receptors on RBCs with their free hemagglutinin binding sites, and RBCs stick together and form aggregates whenever they collide such that virus particles are able to cross-link them.

For virus binding to SA-linked receptors, we assume mass-action kinetics, leading to:

$$
\frac{d V(t)}{d t}=-k_{\mathrm{ass}}^{\mathrm{RBC}} \cdot \underbrace{(1-\theta) \cdot r \cdot V(t)}_{\text {free virus sites }} \cdot \underbrace{[1-\rho(t)] \cdot b \cdot R B C_{\mathrm{tot}_{2}}}_{\text {free RBC sites }}+k_{\mathrm{diss}^{\mathrm{RBC}}}^{\underbrace{\frac{b}{b^{*}} \cdot \rho(t) \cdot R B C_{\mathrm{tot}_{2}}}_{\text {bound RBC sites }}} .
$$


Kinetic constants for association and dissociation are denoted as $k_{\text {ass }}^{\mathrm{RBC}}$ and $k_{\text {diss }}^{\mathrm{RBC}}$. We assume $e=e^{*}=3$ (Poumbourios et al., 1990) to define the contribution of the concentration of hemagglutinin receptors that are not covered by antibodies (free virus sites) to the association rate. Association further depends on the amount of RBC binding sites that are not yet covered by virus, defined by the fraction of covered sites, $\rho(t)$, the average number of SA-linked surface receptors each RBC carries, $b$, and the total concentration of RBCs in step 2 of the assay, $R B C_{\mathrm{tot}_{2}}$. For the dissociation term, we assume that one virus particle covers on average $b^{*}$ binding sites, since influenza virus particles are approximately 60-times smaller than RBCs (see below). The correction by $b^{*}$ reflects the definition of the fraction of covered RBC binding sites (making the term for bound RBS sites equivalent to the concentration of bound virus, $V_{\mathrm{tot}_{2}}-V(t)$ ):

$$
\rho(t)=\frac{b^{*} \cdot\left[V_{\mathrm{tot}_{2}}-V(t)\right]}{b \cdot R B C_{\mathrm{tot}_{2}}} .
$$

To capture RBC aggregation, let $\boldsymbol{B}_{k}$ denote the concentration of agglutinating particles (individual RBCs and RBC aggregates) consisting of $k$ cells, with a maximum aggregate size $N$. To describe the dynamics, we use the Smoluchowski coagulation equation (Von Smoluchowski, 1917), where the rate of agglutination is proportional to an agglutination rate constant $k_{\text {agg }}$ and the number of available cross-linking sites $\rho(t)(1-\rho(t))(1-\theta)^{2}$, which is proportional to the number of mutual pairs of free binding sites on colliding RBCs and can be interpreted as a cross-linking probability:

$$
\frac{d B_{k}(t)}{d t}=k_{\mathrm{agg}} \rho(t)(1-\rho(t))(1-\theta)^{2}\left(\frac{1}{2} \sum_{i+j=k} K_{i j} B_{i}(t) B_{j}(t)-B_{k}(t) \sum_{i=1}^{N} K_{i k} B_{i}(t)\right)
$$

For the special case $K_{i j}=K_{i k}=K$, where the kernel is independent of the particle size, there is a simple analytical solution for the discrete size distribution of aggregates. Let $\sum_{i=1}^{N} B_{i}(t)=$ $B_{N}(t)$ denote the total concentration of particles, and $B_{N}(t=0)=R B C_{\text {tot }_{2}}$ the concentration of particles before agglutination. In addition, from mass conservation follows: $\sum_{k=1}^{N} k B_{k}(t)=$ $R B C_{\mathrm{tot}_{2}}$. Summing over all values of $k$ then yields:

$$
\begin{aligned}
\frac{d B_{N}(t)}{d t} & =k_{\mathrm{agg}} \rho(t)(1-\rho(t))(1-\theta)^{2}\left[\left(\frac{K}{2}\right) \cdot R B C_{\mathrm{tot}_{2}}^{2}-K \cdot R B C_{\mathrm{tot}_{2}}^{2}\right] \\
& =-k_{\mathrm{agg}} \rho(t)(1-\rho(t))(1-\theta)^{2}\left(\frac{K}{2}\right) \cdot R B C_{\mathrm{tot}_{2}}^{2} .
\end{aligned}
$$

Integrating once gives:

$$
B_{N}(t)=\frac{R B C_{\mathrm{tot}_{2}}}{1+\left(\frac{k_{\mathrm{agg}}}{2}\right) \rho(t)(1-\rho(t))(1-\theta)^{2} R B C_{\mathrm{tot}_{2}} t} .
$$

Here, we set $K=1$ such that the effect of $K$ is lumped into $k_{\text {agg }}$ because we estimated $k_{\text {agg }}$ from data (see below). 


\section{Assay step 3: determination of HI titer}

We define the degree of hemagglutination as:

$$
h(t)=\left(1-\frac{B_{N}(t)}{R B C_{\mathrm{tot}_{2}}}\right) \cdot 100,
$$

such that it takes values between $0 \%$ and $100 \%$. If there is no hemagglutination, the concentration of agglutinated particles is the same as the initial concentration of $\operatorname{RBCs}\left(B_{N}(t)=R B C_{\mathrm{tot}_{2}}\right)$ and the degree of hemagglutination is $0 \%$. If all RBCs are agglutinated, there is only one agglutinating particle in the system and $B_{N}(t)=1 / N_{A} \cdot 10^{9} \mathrm{nM}$, where $N_{A}$ is Avogadro's number. Since $N_{A} \approx 6 \cdot 10^{23}, B_{N} \approx 10^{-15} \approx 0 \mathrm{nM}$ such that $h(t)=100 \%$.

\section{Model implementation}

To obtain the degree of hemagglutination $h(t)$ in Equation 5, we compute $\theta$ from Equation 1, $\rho(t)$ for any time point $t$ in assay step 2 from Equations 2 and 3 , and the corresponding $B_{N}(t)$ from Equation 4.

In addition, the total concentration of antibodies is given by

$$
A_{\mathrm{tot}_{1}}=0.5 \cdot d_{p} \cdot d^{j} \cdot A_{0}
$$

where $A_{0}$ is the initial serum antibody concentration, $d_{p}$ is the serum predilution factor, $d$ the serial dilution factor and $j$ the considered dilution step. The total concentrations of virus are

$$
V_{\mathrm{tot}_{1}}=0.5 \cdot V_{0} \text { and } V_{\mathrm{tot}_{2}}=0.5 \cdot V_{\mathrm{tot}_{1}},
$$

because each assay step involves adding equal volumes of solution; $V_{0}$ is the initial virus concentration. Analogously,

$$
R B C_{\mathrm{tot}_{2}}=0.5 \cdot R B C_{0},
$$

where $R B C_{0}$ is the initial concentration of RBCs.

The model is implemented in the R package himodel (https://gitlab.com/csb.ethz/himodel).

\section{Model parameters and initial conditions}

All model parameters and initial conditions could be either extracted or derived from literature (summarized in Table 1), except for the agglutination rate of RBCs $\left(k_{\text {agg }}\right)$, which we estimated from data as described below. 
$R B C$ concentration $\left(R B C_{0}\right)$

Following the WHO protocol (WHO, 2002), a $0.75 \%(\mathrm{v} / \mathrm{v})$ suspension of chicken RBCs is used to measure HI titers against H1N1pdm09. This corresponds approximately to $1.875 \cdot 10^{6}$ cells $/ \mathrm{mL}$ (Tyrrell and Valentine, 1957). Given that $1 \mathrm{~mol}$ corresponds to $6.022 \cdot 10^{23}$ cells, the molar concentration is approximately $R B C_{0}=3.1 \cdot 10^{-5} \mathrm{nM}$. To determine the effect of pipetting errors, we set the RBC concentration range in the sensitivity analysis to $0.375 \%$ $1.5 \%(\mathrm{v} / \mathrm{v})$ suspension, which corresponds to approximately $1.6 \cdot 10^{-5}-6.3 \cdot 10^{-5} \mathrm{nM}$.

\section{Number of sialic acid-linked receptors on $R B C(b)$}

Influenza hemagglutinin binds to SA-linked surface receptors of RBCs. Human H1 influenza viruses bind preferentially to $\alpha 2 \rightarrow 6$ linked SA (Rogers and D'Souza, 1989), which occurs on the surface of chicken RBCs mainly in N-linked glycans (Aich et al., 2011; Aoki, 2017). Chicken RBCs contain a mixture of $\alpha 2 \rightarrow 3$ and $\alpha 2 \rightarrow 6$-linked glycans in a ratio of approximately 60:40 - 50:50 (Aich et al., 2011). The total number of N-glycan on RBCs has been estimated to be $1 \cdot 10^{6}$ (Aoki, 2017). Thus, we assume that the average number of receptors that can interact with hemagglutinin is $0.45 \cdot 10^{6}$. Chicken RBCs also have SA-linked O-glycans such as glycophorins (Duk et al., 2000) on their surface, but most of them contain $\alpha 2 \rightarrow 3$-linked SA. Therefore, we neglect them.

\section{Steric virus factor $\left(b^{*}\right)$ : number of sialic acid-linked receptors covered by bound virions}

Influenza virions are approximately 60-times smaller than RBCs (Harris et al., 2006). To model the binding of virions to RBCs, we need to take into account that bound virions cover multiple SA-linked receptors. We estimated the average number of covered $\alpha 2 \rightarrow 6$ SA-linked receptors, $b^{*}$, from simple geometry. We assume that SA-linked receptors are uniformly distributed on RBCs. Their estimated surface area ranges from $140-160 \mu \mathrm{m}^{2}$ and we assume an average surface area of $A_{\mathrm{RBC}}=150 \cdot 10^{6} \mathrm{~nm}^{2}$ (Ballas, 1987; Movassaghian and Torchilin, 2015). The virus-covered area is determined by the virus' diameter. Most influenza virions are spherical with a diameter ranging from $84-170 \mathrm{~nm}$ and mean diameter $d=120 \mathrm{~nm}$ (Harris et al., 2006). We estimate the shaded area from the circle area, which yields:

$$
b^{*}=\frac{\pi(d / 2)^{2}}{\mathrm{~A}_{\mathrm{RBC}}} \cdot b \approx 34
$$

In the sensitivity analysis, we sample $b^{*}$ assuming $d \sim \operatorname{Unif}(84,170), A_{\mathrm{RBC}} \sim \operatorname{Unif}(130$. $\left.10^{6}, 170 \cdot 10^{6}\right)$, and $b \sim \operatorname{Unif}\left(0.4 \cdot 10^{6}, 0.5 \cdot 10^{6}\right)$, where $d$ has unit nm, $A_{\mathrm{RBC}}$ has unit nm ${ }^{2}$ and $b$ is unitless. 
Virus concentrations $\left(V_{0}\right)$

To ensure the reproducibility of the $\mathrm{HI}$ assay, the same amount of virus particles must be used in each experiment. Therefore, virus concentration is measured in HA units, an operational unit that is determined in the so called HA titration assay, where virus is titrated against a constant amount of RBCs (same amount as used in the HI assay, i.e. $50 \mu \mathrm{L}$ of $0.75 \%$ (v/v) RBC suspension are added to $50 \mu \mathrm{L}$ serum-virus dilution). The amount of virus that agglutinates an equal volume of standardized RBC suspension is defined as 1 HA unit (WHO, 2002). Electron microscopy data show that partial hemagglutination occurs at 1:1 binding (on average, one virus particle binds to one RBC) (Tyrrell and Valentine, 1957). We assume that full hemagglutination requires at least 2:1 binding. We used the rate equation for virus-RBC binding (Equation 2) to determine the virus concentration that leads to $2: 1$ binding with $0.5 \cdot 3.1 \cdot 10^{-5} \mathrm{nM} \mathrm{RBC}$ (Supplementary Figure S1a): $3.2 \cdot 10^{-5} \mathrm{nM}$. Assuming that this virus concentration corresponds to $1 \mathrm{HA}$ unit in our model simulations, $4 \mathrm{HA}$ units are approximately $V_{0}=1.3 \cdot 10^{-3}$ nM. In the sensitivity analysis, we varied $V_{0}$ in the range of $3-7$ HA units.

\section{Agglutination rate $\left(k_{\mathrm{agg}}\right)$}

We inferred the agglutination rate of RBCs from $\mathrm{HI}$ titer and serum IgG concentration of the reference serum using the inference procedure described in the next section. We used a broad uniform prior for $k_{\text {agg }} \sim$ Uniform $\left(10^{5}, 10^{9}\right)$, set the coagulation kernel $K$ to 1 and fixed all remaining parameters to the values in Table 1 . The $k_{\text {agg }}$ posterior distribution was approximately log-normal (centered at around $2 \cdot 10^{6} s^{-1}$ with $95 \%$ credibility interval of approximately $0.4 \cdot 10^{6}$ $-13 \cdot 10^{6} s^{-1}$ ) with slightly heavier tail towards larger $k_{\text {agg }}$ values since hemagglutination reaches saturation at approximately $30 \mathrm{~min}$ (Supplementary Figure S1b). Data at earlier time points would be needed to infer $k_{\text {agg }}$ with higher precision. We set $k_{\text {agg }}=2 \cdot 10^{6} s^{-1}$; the precision suffices as we are interested in hemagglutination at $\geq 30 \mathrm{~min}$.

\section{Inference of neutralizing antibody avidities}

Given a measured $\operatorname{IgG}$ antibody concentration $A_{i}$ of serum sample $i$ (with estimated log mean $\mu_{A, i}$ and log standard deviation $\sigma_{A, i}$ ) and the corresponding HI titer determined in an HI assay with $j=1, \ldots, J$ dilution steps, serum predilution factor $d_{p}$, and serial dilution factor $d$, the generative model to infer the posterior distributions for $K_{D, i}^{\text {app }}$ is defined as follows: 


$$
\mathrm{P}\left(K_{\mathrm{D}, i}^{\mathrm{app}}, A_{i} \mid \mathbf{y}_{\mathbf{i}}\right)=\frac{\mathrm{P}\left(K_{\mathrm{D}, i}^{\mathrm{app}}\right) \mathrm{P}\left(A_{i}\right) \mathrm{P}\left(\mathbf{y}_{\mathbf{i}} \mid K_{\mathrm{D}, i}^{\mathrm{app}}, A_{i}\right)}{\mathrm{P}\left(\mathbf{y}_{\mathbf{i}}\right)} .
$$


We sampled posterior distributions using the Metropolis-Hastings algorithm (Brooks et al., 2011) with 6000 samples, burn-in size of 1000 samples, and 5 chains. We used a broad lognormal prior for $K_{\mathrm{D}, i}^{\mathrm{app}}$ centered at $1 \mathrm{nM}$ with $\log$ mean $\mu_{k}=0$ and $\log$ standard deviation $\sigma_{K}=4$. To define the value of $h_{0}$, we investigated the relationship between HA units and hemagglutination degree in our HA titration simulations. The model predicted that the hemagglutination degree is $>75 \%$ for $\geq 1$ HA unit (Supplementary Figure S1c), which by definition corresponds to full hemagglutination. Thus, assuming symmetry, we consider $h_{0}=25 \%$ a reasonable estimate, also assuming that differences below $25 \%$ cannot be distinguished by eye. However, a different value for $h_{0}$ does not affect the interpretation of our results: it would only shift the maximum a posteriori (MAP) estimates of all samples either towards lower avidities (for larger $h_{0}$ ) or higher avidities (for smaller $h_{0}$ ). Steepness parameter $\alpha$ does not affect the MAP estimate, but only the width of the posterior distribution. Here, we set $\alpha=15$ and then investigated the relationship between posterior distribution and resulting $\mathrm{HI}$ titer by sampling. We sampled 500 times from the joint posterior distribution of $K_{\mathrm{D}, i}^{\mathrm{app}}$ and $A_{i}$ for all patient sera $i$ and predicted the resulting $\mathrm{HI}$ titer to investigate the uncertainty in $K_{\mathrm{D}, i}^{\mathrm{app}}$ due to discretization of $\mathrm{HI}$ titer measurements. On average, approximately $55 \%$ of samples resulted in the observed $\mathrm{HI}$ titer, whereas approximately $95 \%$ of samples also included HI titers one dilution step higher or lower than the actually observed HI titer.

\section{Sensitivity analysis}

Sobol sensitivity analysis attributes variance in model output to individual model input factors using variance decomposition (Saltelli et al., 2004). Given $k$ model inputs, the total variance $V(y)$ in model output can be decomposed as:

$$
V(y)=\sum_{i} V_{i}+\sum_{i} \sum_{j>i} V_{i j}+\ldots+V_{12 \ldots k}
$$

where $V_{i}=V\left(E\left(Y \mid x_{i}\right)\right)$ is the variance with respect to the distribution of input factor $x_{i}$. The second-order interaction term $V_{i j}=V\left(E\left(Y \mid x_{i}, x_{j}\right)\right)-V_{i}-V_{j}$ captures the part of the effect of $x_{i}$ and $x_{j}$ that is not described by the first order terms $V_{i}, V_{j}$ and so on. The relative contribution of each term to the unconditional variance $V(y)$ serves as a measure of sensitivity. For instance, $V_{i}$ will be large, if $x_{i}$ is influential. The first order Sobol sensitivity index is defined as

$$
S_{i}=\frac{V_{i}}{V(y)}
$$

To obtain the total contribution of $x_{i}$, that is the sum of all terms in the variance decomposition that include $x_{i}$, we compute the total contribution to variance $V(y)$ due to all factors but $x_{i}$, denoted by $\mathbf{x}_{-\mathbf{1}}$. The total Sobol sensitivity index for $x_{i}$ is then given by

$$
S_{i}^{T}=\frac{V(y)-V\left(E\left(y \mid \mathbf{x}_{-1}\right)\right)}{V(y)} .
$$


We used Monte Carlo estimation to estimate Sobol indices (Jansen, 1999; Saltelli et al., 2010) implemented in the R package sensitivity (Iooss et al., 2019) with $n=10000$ random samples of model input vector $\mathbf{x}^{\mathbf{T}}=\left(x_{1}, x_{2}, \ldots, x_{k}\right)$ and 10 bootstrap replicates to estimate confidence intervals. Input variables were assumed to be independent of each other. We considered $k=12$ inputs sampled within a biologically reasonable range (Table 1).

\section{Ethics statement}

The study was conducted in accordance with the Declaration of Helsinki and approved by the Ethic committee northwest and central Switzerland (EKNZ ID 2014-141). All patients signed informed consent.

\section{Patient sera}

Adult patients that received allogeneic hematopoietic stem cell transplantation (HSCT) at least one year before were recruited in a multicenter cohort study at three hematological centers in Switzerland (at the University Hospital Basel and the Cantonal Hospitals in Aarau and Lucerne) between October 2014 and January 2015. Only patients without known vaccine intolerance such as egg protein allergy or vaccine-associated adverse events were eligible for participation. In total, 57 patients were recruited; we included 45 of them in the present study based on the availability of serum samples. Following the standard of care for HSCT patients, each patient received two doses of non-adjuvanted trivalent influenza vaccine (TIV), where the second dose was given 30 days after the first. Serum samples were collected prior to first vaccination (d0) and after vaccination $(\mathrm{d} 7, \mathrm{~d} 30, \mathrm{~d} 60, \mathrm{~d} 180)$ and stored in aliquots at $-80^{\circ} \mathrm{C}$. Almost all patients were in complete remission $(42 / 45,93 \%)$, and no patient showed progression. In the present study, 16 patients (36\%) were within the first two years after HSC transplantation. 20 patients (44\%) showed seroprotective titers against H1N1pmd09 before vaccination. As classified by the treating physician, 26 (60\%) were immunosuppressed, of which 7 (16\%) were classified with severe immunosuppression (grade 3). According to NIH consensus criteria (Filipovich et al., 2005), 30 patients suffered from chronic GVHD, of which 11 (24\%) were classified with severe chronic GVHD (grade 3). An overview of patient's baseline characteristics is given in Table 2.

\section{Vaccine composition}

Patients received two doses of the non-adjuvanted 2014/2015 trivalent influenza vaccine (Agrippal, Novartis, Switzerland), comprising inactivated, subunit influenza virus with $15 \mu \mathrm{g}$ HA antigen of each vaccine strain: A/California/7/2009 (H1N1pdm09), A/Texas/50/2012 (H3N2) and B/Massachusetts/2/2012 (Yamagata lineage). 
Table 2. Characteristics of allogeneic hematopoietic stem cell transplant patients (all patients and subset of patients with experimentally determined avidities for validation of inferred avidities).

\begin{tabular}{|c|c|c|c|}
\hline & & All & Validation subset \\
\hline Total & & 45 & 12 \\
\hline \multirow[t]{2}{*}{ Age } & Median, IQR (years) & $58,44-64$ & $45,43-67$ \\
\hline & $\geq 65$ years & $11(25 \%)$ & $4(33 \%)$ \\
\hline \multirow[t]{2}{*}{ Sex } & Male & $23(51 \%)$ & $4(33 \%)$ \\
\hline & Female & $22(49 \%)$ & $8(67 \%)$ \\
\hline \multirow[t]{9}{*}{ Underlying disease } & Acute myeloid leukemia (AML) & $17(38 \%)$ & $6(50 \%)$ \\
\hline & Acute lymphoblastic leukemia (ALL) & $8(18 \%)$ & $2(17 \%)$ \\
\hline & Chronic myeloid leukemia (CML) & $5(11 \%)$ & $1(8 \%)$ \\
\hline & Chronic lymphocytic leukemia (CLL) & $5(11 \%)$ & $1(8 \%)$ \\
\hline & Multiple myeloma (MM) & $5(11 \%)$ & $1(8 \%)$ \\
\hline & Plasma cell leukemia (PCL) & $1(2 \%)$ & 0 \\
\hline & Myeloproliferative neoplasms (MPN) & $1(2 \%)$ & $1(8 \%)$ \\
\hline & Myelodysplastic syndromes (MDS) & $2(4 \%)$ & 0 \\
\hline & Non-Hodgkin lymphoma (NHL) & $1(2 \%)$ & 0 \\
\hline \multirow{4}{*}{$\begin{array}{l}\text { Time after } \\
\text { transplantation }\end{array}$} & median, IQR (years) & $4,2-8$ & $6,3-8$ \\
\hline & $1-2$ years & $16(36 \%)$ & $2(17 \%)$ \\
\hline & $3-5$ years & $13(29 \%)$ & $3(25 \%)$ \\
\hline & $>5$ years & $16(36 \%)$ & $7(58 \%)$ \\
\hline \multirow[t]{2}{*}{ Transplant source } & Peripheral blood & $40(89 \%)$ & $11(92 \%)$ \\
\hline & Bone marrow & $5(11 \%)$ & $1(8 \%)$ \\
\hline \multirow[t]{2}{*}{ Donor source } & Matched related donor & $16(36 \%)$ & $5(42 \%)$ \\
\hline & Matched unrelated donor & $21(47 \%)$ & $5(42 \%)$ \\
\hline \multirow[t]{4}{*}{ Disease status $^{\mathrm{a}}$} & Complete remission & $42(93 \%)$ & $12(100 \%)$ \\
\hline & Stable & $1(2 \%)$ & 0 \\
\hline & Recurrence & $2(4 \%)$ & 0 \\
\hline & Progressive & 0 & 0 \\
\hline \multirow[t]{4}{*}{ Immunosuppression $^{\mathrm{a}}$} & None & $18(40 \%)$ & $2(17 \%)$ \\
\hline & Mild (grade 1) & $6(13 \%)$ & $2(17 \%)$ \\
\hline & Moderate (grade 2) & $14(31 \%)$ & $6(50 \%)$ \\
\hline & Severe (grade 3) & $7(16 \%)$ & $2(17 \%)$ \\
\hline Immunosuppressive & Prednisone & $13(29 \%)$ & $5(42 \%)$ \\
\hline \multirow[t]{4}{*}{ treatment $^{\mathrm{a}}$} & Tacrolimus & $14(31 \%)$ & $7(58 \%)$ \\
\hline & Mycophenolate $^{b}$ & $9(20 \%)$ & $3(25 \%)$ \\
\hline & Cyclosporine $\mathrm{A}^{\mathrm{c}}$ & $4(9 \%)$ & 0 \\
\hline & Rituximab $^{\mathrm{d}}$ & $3(7 \%)$ & 0 \\
\hline \multirow[t]{4}{*}{ Chronic GVHD } & None & $15(33 \%)$ & 0 \\
\hline & Mild (grade 1) & $9(20 \%)$ & $4(33 \%)$ \\
\hline & Moderate (grade 2) & $10(22 \%)$ & $6(50 \%)$ \\
\hline & Severe (grade 3) & $11(24 \%)$ & $2(17 \%)$ \\
\hline
\end{tabular}




\section{HI assay}

$\mathrm{HI}$ assays were performed according to the WHO manual (WHO, 2002). Sera were pre-treated with receptor destroying enzyme (RDE); Sigma-Aldrich, C8772) and two-fold serially diluted, covering dilutions from 1:8 to 1:2048. A 0.75\% (v/v) suspension of chicken RBCs (Cedarlane, CLC8800) and 4 HA units of influenza H1N1pdm09 virus (NYMC-X181) were used to perform the assay. The reported $\mathrm{HI}$ titer is the dilution factor of the highest serum dilution that showed full hemagglutination inhibition. The protocol has been published in detail (Kaufmann et al., 2017).

\section{ELISA for influenza-specific IgG detection}

ELISA 96-well plates (Thermo Scientific, 442404) were coated with $0.5 \mu \mathrm{g} / \mathrm{mL}$ whole virus H1N1pdm09 (NYMC-X181, $45 \mu \mathrm{g} \mathrm{HA} / \mathrm{mL}$ ) at $4^{\circ} \mathrm{C}$ overnight. Plates were blocked with 5\% bovine serum albumin (BSA) in PBS for $1 \mathrm{~h}$ at room temperature (RT). Patient serum samples were 1:4000 diluted in 0.5\% BSA in PBS. Reference serum was 1:1000 diluted (top dilution of calibration curve) and then six times four-fold serially diluted, yielding a calibration curve with seven measurements. After blocking and washing with 0.05\% TWEEN 20 in PBS, 100 $\mu \mathrm{L} / w e l l$ of diluted serum samples were added and incubated for $2 \mathrm{~h}$ at RT. Unbound serum antibodies were removed by washing the plates four times, and bound serum $\operatorname{IgG}$ was detected by $70 \mu \mathrm{L} /$ well of 1:3000 diluted rabbit anti-human $\mathrm{IgG}$ antibody linked to horseradish peroxidase (Agilent, P021402-2) incubated for 2h at RT. After washing, plates were developed with $100 \mu \mathrm{L} /$ well TMB substrate solution (BD, 555214) for $15 \mathrm{~min}$ and stopped with $50 \mu \mathrm{L} /$ well $2 \mathrm{~N} \mathrm{H}_{2} \mathrm{SO}_{4}$. Absorbance was measured at 450 and $620 \mathrm{~nm}$. Measurements were backgroundand blank-corrected. To obtain a calibration curve, reference measurements were fitted using a four-parameter logistic equation (log concentration vs log absorbance). All measurements were performed in duplicates.

\section{Urea elution assay to measure IgG avidities}

The ELISA assay described above was adapted to measure serum $\operatorname{IgG}$ avidities against influenza H1N1pdm09. Each serum was accordingly diluted to obtain a final concentration within the linear range of the calibration curve. After incubation with serum and washing as described above, each well was incubated for an additional $3 \mathrm{~h}$ at RT with either $100 \mu \mathrm{L} /$ well $4 \mathrm{M}$ urea (treated) or $100 \mu \mathrm{L} /$ well PBS (untreated). The concentration of bound IgG was determined using a calibration curve, as described above. The fraction of IgG remaining bound after urea treatment compared to the untreated wells is reported as the avidity index. Avidities were determined in two experiments, each performed in duplicates. 


\section{Reference serum}

The concentration of H1N1pdm09-specific IgG antibodies was determined in ELISA experiments relative to a reference serum collected from a healthy volunteer on day 7 after vaccination with 2014/2015 TIV (Agrippal, Novartis, Switzerland), showing an HI titer of 512. Since the absolute reference concentration could only be determined by mass spectrometry, which was not feasible in this study, we estimated the concentration based on reported H1N1pdm09-specific IgG concentrations in vaccinated healthy adults with similar HI titers (Eidem et al., 2015). We set the reference concentration to $100 \mu \mathrm{g} / \mathrm{mL}(670 \mathrm{nM})$, yielding an estimated avidity for the reference serum of $0.4-0.8 \mathrm{nM}$, consistent with observed affinities for post-vaccination serum IgG for H1N1pdm09 in healthy adults (Lee et al., 2016).

\section{Identification of patients with increase in avidity and increase in non-neutralizing IgG}

For each inferred $K_{D}^{\text {app }}$ value, we identified the uncertainty interval due to ELISA measurement error and dichotomization in HI titers by sampling from the joint posterior distribution (see above) and considered non-overlapping intervals as a significant change in $K_{D}^{\text {app }}$. To detect patients that produced non-neutralizing IgG after vaccination, we identified patients that showed no increase in $\mathrm{HI}$ titer while showing an increase in serum IgG that resulted in a significant decrease in avidity (Supplementary Figure S4a).

\section{Statistical analysis}

Serum IgG and inferred $K_{D}^{\text {app }}$ values were available for 43 patients at five time points $(t=$ $0,7,30,60,180$ days) with 197 observations in total. To estimate the effects of a patient's immune state on serum IgG and avidity $\left(1 / K_{D}^{\text {app }}\right)$, we used a linear mixed model with patientspecific random intercepts that takes the following general form:

$$
\begin{aligned}
y_{i j} & =\beta_{0}+x_{i j}^{T} \beta_{1}+\gamma_{i}+\epsilon_{i j}, \\
\gamma_{i} & \sim \mathcal{N}\left(0, \sigma_{\gamma}^{2}\right), \\
\epsilon_{i j} & \sim \mathcal{N}\left(0, \sigma_{\epsilon}^{2}\right),
\end{aligned}
$$

where $y_{i j}$ is the $\log 2$-transformed $\operatorname{IgG}$ concentration or $1 / K_{D}^{\text {app }}$ value, respectively, of patient $i$ at time point $j, x_{i j}$ is a $p$-dimensional vector of $p$ covariates, $\beta_{0}$ is an intercept term, $\beta_{1}$ is a vector of fixed effects, $\gamma_{i}$ the random patient-specific intercept, and $\epsilon_{i j}$ models the withinpatient measurement error. We modeled the observed rise and fall of serum $\operatorname{IgG}$ and $1 / K_{D}^{\text {app }}$ value after vaccination using a second-degree polynomial. To distinguish time trends in avidity between neutralizing and non-neutralizing IgG responders, we added a dummy variable for 
neutralizing response when analyzing response in avidity. Time post HSCT $\leq 2$ years, cGVHD grade, and immunosuppression grade were added as fixed effects on intercept to investigate effects on baseline, and on slope to investigate effects on response. Time post HSCT $\leq 2$ years was encoded as a binary variable ( 1 for $\leq 2$ years, 0 for $>2$ years). Both cGVHD and immunosuppression grade were encoded as numerical variables with values $0,1,2,3$, such that grade 0 is the reference level, and there is a linear increase in effect with increasing grade. To control for potential confounders, we corrected for sex and age. For model selection, the full model with fixed effects on slope and intercept was fitted using maximum likelihood estimation implemented in the lmer4 package (Bates et al., 2015) and type II ANOVA by Satterthwaite's approximation provided by the lmerTest package (Kuznetsova et al., 2017).

We detected no significant effects on response for serum IgG and avidity. Therefore, we removed fixed effects on slope and refitted the final models using restricted maximum likelihood estimation to obtain unbiased estimates (Bates et al., 2015). Residuals indicated that the normality assumption was satisfied (Supplementary Figure S4b). Confidence intervals were computed via the Wald method provided by lme4. To compare the results with HI titers, we estimated the effect of time post HSCT $\leq 2$ years, cGVHD grade, and immunosuppression on HI titers controlling for age, sex, and time after vaccination using a generalized linear regression model for sequential, ordered data (Tutz, 1991). The model was fitted using maximum likelihood estimation implemented in VGAM (Yee et al., 2010).

\section{Data and software availability}

The model is available in the R package himodel (https://gitlab.com/csb.ethz/himodel). Source files necessary to reproduce the results of this work are available on GitLab (https://gitlab.com/csb.ethz/himodel-manuscript).

\section{Conflict of interests}

None.

\section{Author contributions}

JL, JS, and AE designed the study. JL and JS developed the model. JL and MS designed and performed experiments. JL analyzed data, implemented the model, and performed simulations. $\mathrm{JH}$ and $\mathrm{YH}$ recruited patients, collected patient samples, and patient information. $\mathrm{YH}, \mathrm{JH}$ and AE consulted on medical questions. JS and AE supervised the project. JL and JS wrote the first draft of the manuscript, and all authors commented on the final version. 


\section{Acknowledgments}

We thank our clinical collaborators Nathan Cantoni (Cantonal Hospital Aarau), and Sabine Ruosch (Cantonal Hospital Lucerne) for providing patient sera and supporting the cohort study. We thank Dominik Vogt and Lukas Kaufmann for technical support with patient samples and Fabian Rudolf for advice on the experimental design of avidity measurements.

\section{References}

U. Aich, N. Beckley, Z. Shriver, R. Raman, K. Viswanathan, S. Hobbie, and R. Sasisekharan. Glycomics-based analysis of chicken red blood cells provides insight into the selectivity of the viral agglutination assay. The FEBS journal, 278(10):1699-1712, 2011.

M. O. Altman, J. R. Bennink, J. W. Yewdell, and B. R. Herrin. Lamprey VLRB response to influenza virus supports universal rules of immunogenicity and antigenicity. Elife, 4:e07467, 2015 .

D. Angeletti, J. S. Gibbs, M. Angel, I. Kosik, H. D. Hickman, G. M. Frank, S. R. Das, A. K. Wheatley, M. Prabhakaran, D. J. Leggat, et al. Defining B cell immunodominance to viruses. Nature immunology, 18(4):456, 2017.

T. Aoki. A comprehensive review of our current understanding of red blood cell (RBC) glycoproteins. Membranes, 7(4):56, 2017.

P. Arevalo, H. Q. McLean, E. A. Belongia, and S. Cobey. Earliest infections predict the age distribution of seasonal influenza a cases. Elife, 9:e50060, 2020.

S. K. Ballas. Erythrocyte concentration and volume are inversely related. Clinica chimica acta; international journal of clinical chemistry, 164(2):243-244, 1987.

D. Bates, M. Mächler, B. Bolker, and S. Walker. Fitting linear mixed-effects models using lme4. Journal of Statistical Software, 67(1):1-48, 2015. doi: 10.18637/jss.v067.i01.

A. Benoit, J. Beran, J.-M. Devaster, M. Esen, O. Launay, G. Leroux-Roels, J. E. McElhaney, L. Oostvogels, G. A. van Essen, M. Gaglani, et al. Hemagglutination inhibition antibody titers as a correlate of protection against seasonal A/H3N2 influenza disease. In Open forum infectious diseases, volume 2, page ofv067. Oxford University Press, 2015.

C. Berek, A. Berger, and M. Apel. Maturation of the immune response in germinal centers. Cell, 67(6):1121-1129, 1991.

S. Brooks, A. Gelman, G. Jones, and X.-L. Meng. Handbook of markov chain monte carlo. CRC press, 2011.

G. W. Brunette, P. E. Kozarsky, and N. J. Cohen. CDC health information for international travel 2016: the yellow book. Oxford University Press, USA, 2015. 
N. S. De Silva and U. Klein. Dynamics of B cells in germinal centres. Nature reviews immunology, 15(3):137-148, 2015.

E. B. Dolgosheina, A. Y. Karulin, and A. V. Bobylev. A kinetic model of the agglutination process. Mathematical biosciences, 109(1):1-10, 1992.

M. Duk, H. Krotkiewski, T. V. Stasyk, M. Lutsik-Kordovsky, D. Syper, and E. Lisowska. Isolation and characterization of glycophorin from nucleated (chicken) erythrocytes. Archives of biochemistry and biophysics, 375(1):111-118, 2000.

M. Edwards and N. Dimmock. Two influenza A virus-specific Fabs neutralize by inhibiting virus attachment to target cells, while neutralization by their IgGs is complex and occurs simultaneously through fusion inhibition and attachment inhibition. Virology, 278(2):423435, 2000.

S. Eidem, S. M. Tete, Å. Jul-Larsen, K. Hoschler, E. Montomoli, K. A. Brokstad, and R. J. Cox. Persistence and avidity maturation of antibodies to A (H1N1) pdm09 in healthcare workers following repeated annual vaccinations. Vaccine, 33(33):4146-4154, 2015.

A. H. Ellebedy. Immunizing the immune: can we overcome influenza's most formidable challenge? Vaccines, 6(4):68, 2018.

D. Engelhard, Z. Zakay-Rones, M. Y. Shapira, I. Resnick, D. Averbuch, S. Grisariu, L. Dray, E. Djian, N. Strauss-Liviatan, I. Grotto, et al. The humoral immune response of hematopoietic stem cell transplantation recipients to AS03-adjuvanted A/California/7/2009 (H1N1) v-like virus vaccine during the 2009 pandemic. Vaccine, 29(9):1777-1782, 2011.

A. H. Filipovich, D. Weisdorf, S. Pavletic, G. Socie, J. R. Wingard, S. J. Lee, P. Martin, J. Chien, D. Przepiorka, D. Couriel, et al. National Institutes of Health consensus development project on criteria for clinical trials in chronic graft-versus-host disease: I. Diagnosis and staging working group report. Biology of blood and marrow transplantation, 11(12):945-956, 2005.

G. M. Frank, D. Angeletti, W. L. Ince, J. S. Gibbs, S. Khurana, A. K. Wheatley, E. E. Max, A. B. McDermott, H. Golding, J. Stevens, et al. A simple flow-cytometric method measuring b cell surface immunoglobulin avidity enables characterization of affinity maturation to influenza A virus. MBio, 6(4):e01156-15, 2015.

W. Gerhard, J. Yewdell, M. E. Frankel, and R. Webster. Antigenic structure of influenza virus haemagglutinin defined by hybridoma antibodies. Nature, 290(5808):713, 1981.

A. Gonzalez-Quintela, R. Alende, F. Gude, J. Campos, J. Rey, L. Meijide, C. Fernandez-Merino, and C. Vidal. Serum levels of immunoglobulins (IgG, IgA, IgM) in a general adult population and their relationship with alcohol consumption, smoking and common metabolic abnormalities. Clinical \& Experimental Immunology, 151(1):42-50, 2008. 
H. T. Greinix, D. Pohlreich, M. Kouba, U. Körmöczi, I. Lohmann, K. Feldmann, C. Zielinski, and W. F. Pickl. Elevated numbers of immature/transitional CD21- B lymphocytes and deficiency of memory CD27+ B cells identify patients with active chronic graft-versus-host disease. Biology of Blood and Marrow Transplantation, 14(2):208-219, 2008.

S. F. d. S. Groth. The neutralization of viruses. In Advances in Virus Research, volume 9, pages 1-125. Elsevier, 1963.

S. Gueller, R. Allwinn, S. Mousset, H. Martin, I. Wieters, E. Herrmann, H. Serve, M. Bickel, and G. Bug. Enhanced immune response after a second dose of an AS03-adjuvanted H1N1 influenza A vaccine in patients after hematopoietic stem cell transplantation. Biology of Blood and Marrow Transplantation, 17(10):1546-1550, 2011.

A. Harris, G. Cardone, D. C. Winkler, J. B. Heymann, M. Brecher, J. M. White, and A. C. Steven. Influenza virus pleiomorphy characterized by cryoelectron tomography. Proceedings of the National Academy of Sciences, 103(50):19123-19127, 2006.

A. K. Harris, J. R. Meyerson, Y. Matsuoka, O. Kuybeda, A. Moran, D. Bliss, S. R. Das, J. W. Yewdell, G. Sapiro, K. Subbarao, et al. Structure and accessibility of HA trimers on intact 2009 H1N1 pandemic influenza virus to stem region-specific neutralizing antibodies. Proceedings of the National Academy of Sciences, 110(12):4592-4597, 2013.

I. Hilgendorf, M. Freund, W. Jilg, H. Einsele, J. Gea-Banacloche, H. Greinix, J. Halter, A. Lawitschka, D. Wolff, and R. Meisel. Vaccination of allogeneic haematopoietic stem cell transplant recipients: report from the international consensus conference on clinical practice in chronic GVHD. Vaccine, 29(16):2825-2833, 2011.

G. K. Hirst. The agglutination of red cells by allantoic fluid of chick embryos infected with influenza virus. Science, 94(2427):22-23, 1941.

K. B. Hoehn, J. S. Turner, F. I. Miller, R. Jiang, O. G. Pybus, A. H. Ellebedy, and S. H. Kleinstein. Human b cell lineages engaged by germinal centers following influenza vaccination are measurably evolving. bioRxiv, 2021.

B. Iooss, A. Janon, G. Pujol, with contributions from Baptiste Broto, K. Boumhaout, S. D. Veiga, T. Delage, J. Fruth, L. Gilquin, J. Guillaume, L. Le Gratiet, P. Lemaitre, A. Marrel, A. Meynaoui, B. L. Nelson, F. Monari, R. Oomen, O. Rakovec, B. Ramos, O. Roustant, E. Song, J. Staum, R. Sueur, T. Touati, and F. Weber. sensitivity: Global Sensitivity Analysis of Model Outputs, 2019. URL https://CRAN.R-project.org/package=sensitivity. R package version 1.16.2.

N. C. Issa, F. M. Marty, L. S. Gagne, S. Koo, K. A. Verrill, E. P. Alyea, C. S. Cutler, J. Koreth, P. Armand, V. T. Ho, et al. Seroprotective titers against 2009 H1N1 influenza A virus after vaccination in allogeneic hematopoietic stem cell transplantation recipients. Biology of Blood and Marrow Transplantation, 17(3):434-438, 2011. 
D. Jackson, B. Crabb, P. Poumbourios, W. Tulip, and W. Laver. Three antibody molecules can bind simultaneously to each monomer of the tetramer of influenza virus neuraminidase and the trimer of influenza virus hemagglutinin. Archives of virology, 116(1-4):45-56, 1991.

C. A. Jacobson, L. Sun, H. T. Kim, S. M. McDonough, C. G. Reynolds, M. Schowalter, J. Koreth, C. S. Cutler, V. T. Ho, E. P. Alyea, et al. Post-transplantation B cell activating factor and B cell recovery before onset of chronic graft-versus-host disease. Biology of Blood and Marrow Transplantation, 20(5):668-675, 2014.

M. J. Jansen. Analysis of variance designs for model output. Computer Physics Communications, 117(1-2):35-43, 1999.

L. Kaufmann, M. Syedbasha, D. Vogt, Y. Hollenstein, J. Hartmann, J. E. Linnik, and A. Egli. An optimized hemagglutination inhibition (HI) assay to quantify influenza-specific antibody titers. JoVE (Journal of Visualized Experiments), (130):e55833, 2017.

S. Khurana, N. Verma, J. W. Yewdell, A. K. Hilbert, F. Castellino, M. Lattanzi, G. Del Giudice, R. Rappuoli, and H. Golding. MF59 adjuvant enhances diversity and affinity of antibodymediated immune response to pandemic influenza vaccines. Science translational medicine, 3(85):85ra48-85ra48, 2011.

S. Khurana, D. Frasca, B. Blomberg, and H. Golding. AID activity in B cells strongly correlates with polyclonal antibody affinity maturation in-vivo following pandemic 2009-H1N1 vaccination in humans. PLoS pathogens, 8(9), 2012.

S. Khurana, M. Hahn, E. M. Coyle, L. R. King, T.-L. Lin, J. Treanor, A. Sant, and H. Golding. Repeat vaccination reduces antibody affinity maturation across different influenza vaccine platforms in humans. Nature communications, 10(1):3338, 2019.

F. Krammer. Novel universal influenza virus vaccine approaches. Current opinion in virology, 17:95-103, 2016.

F. Krammer. The human antibody response to influenza a virus infection and vaccination. Nature Reviews Immunology, 19(6):383-397, 2019.

K. M. Kunisaki and E. N. Janoff. Influenza in immunosuppressed populations: a review of infection frequency, morbidity, mortality, and vaccine responses. The Lancet infectious diseases, 9(8):493-504, 2009.

A. Kuznetsova, P. B. Brockhoff, and R. H. B. Christensen. lmerTest package: Tests in linear mixed effects models. Journal of Statistical Software, 82(13):1-26, 2017. doi: 10.18637/jss. v082.i13.

N. Kylilis, P. Riangrungroj, H.-E. Lai, V. Salema, L. A. Fernandez, G.-B. V. Stan, P. S. Freemont, and K. M. Polizzi. Whole-cell biosensor with tunable limit of detection enables low-cost agglutination assays for medical diagnostic applications. ACS sensors, 4(2):370$378,2019$. 
J. Lee, D. R. Boutz, V. Chromikova, M. G. Joyce, C. Vollmers, K. Leung, A. P. Horton, B. J. DeKosky, C.-H. Lee, J. J. Lavinder, et al. Molecular-level analysis of the serum antibody repertoire in young adults before and after seasonal influenza vaccination. Nature medicine, 22(12):1456, 2016.

Z.-N. Li, S.-C. Lin, P. J. Carney, J. Li, F. Liu, X. Lu, M. Liu, J. Stevens, M. Levine, J. M. Katz, et al. IgM, IgG, and IgA antibody responses to influenza A (H1N1) pdm09 hemagglutinin in infected persons during the first wave of the 2009 pandemic in the United States. Clin. Vaccine Immunol., 21(8):1054-1060, 2014.

M. Meyer-Hermann. Injection of antibodies against immunodominant epitopes tunes germinal centers to generate broadly neutralizing antibodies. Cell reports, 29(5):1066-1073, 2019.

T. K. Ming, H. S. Goodman, and B. BROWN. Mathematical model for the process of aggregation in immune agglutination. Nature, 208(5005):84, 1965.

B. Mohty, M. Bel, M. Vukicevic, M. Nagy, E. Levrat, S. Meier, S. Grillet, C. Combescure, L. Kaiser, Y. Chalandon, et al. Graft-versus-host disease is the major determinant of humoral responses to the AS03-adjuvanted influenza A/09/H1N1 vaccine in allogeneic hematopoietic stem cell transplant recipients. haematologica, 96(6):896-904, 2011.

S. Movassaghian and V. P. Torchilin. Long-circulating therapies for cancer treatment. In Novel Approaches and Strategies for Biologics, Vaccines and Cancer Therapies, pages 433-462. Elsevier, 2015.

J. Ogonek, M. Kralj Juric, S. Ghimire, P. R. Varanasi, E. Holler, H. Greinix, and E. Weissinger. Immune reconstitution after allogeneic hematopoietic stem cell transplantation. Frontiers in immunology, 7:507, 2016.

J. Olsson, J. Johansson, E. Honkala, B. Blomqvist, E. Kok, B. Weidung, H. Lövheim, and F. Elgh. Urea dilution of serum for reproducible anti-HSV1 IgG avidity index. BMC infectious diseases, 19(1):164, 2019.

W. Olszewska, O. E. Obeid, and M. W. Steward. Protection against measles virus-induced encephalitis by anti-mimotope antibodies: the role of antibody affinity. Virology, 272(1): 98-105, 2000.

J. J. Otterstrom, B. Brandenburg, M. H. Koldijk, J. Juraszek, C. Tang, S. Mashaghi, T. Kwaks, J. Goudsmit, R. Vogels, R. H. Friesen, et al. Relating influenza virus membrane fusion kinetics to stoichiometry of neutralizing antibodies at the single-particle level. Proceedings of the National Academy of Sciences, 111(48):E5143-E5148, 2014.

A. Palache, W. Beyer, G. Rimmelzwaan, A. Boon, A. Osterhaus, et al. Haemagglutinationinhibiting antibody to influenza virus. Developments in biologicals, 115:63-73, 2003. 
F. P. Polack, S. J. Hoffman, G. Crujeiras, and D. E. Griffin. A role for nonprotective complementfixing antibodies with low avidity for measles virus in atypical measles. Nature medicine, 9 (9):1209-1213, 2003.

P. Poumbourios, L. E. Brown, D. O. White, and D. C. Jackson. The stoichiometry of binding between monoclonal antibody molecules and the hemagglutinin of influenza virus. Virology, 179(2):768-776, 1990.

G. N. Rogers and B. L. D'Souza. Receptor binding properties of human and animal h1 influenza virus isolates. Virology, 173(1):317-322, 1989.

D. Roll, J. Ammer, B. Holler, B. Salzberger, B. Schweiger, W. Jilg, R. Andreesen, M. Edinger, D. Wolff, and E. Holler. Vaccination against pandemic H1N1 (2009) in patients after allogeneic hematopoietic stem cell transplantation: a retrospective analysis. Infection, 40(2): 153-161, 2012.

R. Ruigrok, P. Andree, R. H. Van Huysduynen, and J. Mellema. Characterization of three highly purified influenza virus strains by electron microscopy. Journal of general virology, 65(4): 799-802, 1984.

A. Saltelli, S. Tarantola, F. Campolongo, and M. Ratto. Sensitivity analysis in practice: a guide to assessing scientific models, volume 1. Wiley Online Library, 2004.

A. Saltelli, P. Annoni, I. Azzini, F. Campolongo, M. Ratto, and S. Tarantola. Variance based sensitivity analysis of model output. design and estimator for the total sensitivity index. Computer Physics Communications, 181(2):259-270, 2010.

S. Sarantopoulos, K. E. Stevenson, H. T. Kim, N. S. Bhuiya, C. S. Cutler, R. J. Soiffer, J. H. Antin, and J. Ritz. High levels of B-cell activating factor in patients with active chronic graft-versus-host disease. Clinical Cancer Research, 13(20):6107-6114, 2007.

S. Sarantopoulos, K. E. Stevenson, H. T. Kim, C. S. Cutler, N. S. Bhuiya, M. Schowalter, V. T. Ho, E. P. Alyea, J. Koreth, B. R. Blazar, et al. Altered B-cell homeostasis and excess BAFF in human chronic graft-versus-host disease. Blood, The Journal of the American Society of Hematology, 113(16):3865-3874, 2009.

N. K. Sauter, M. D. Bednarski, B. A. Wurzburg, J. E. Hanson, G. M. Whitesides, J. J. Skehel, and D. C. Wiley. Hemagglutinins from two influenza virus variants bind to sialic acid derivatives with millimolar dissociation constants: a 500-MHz proton nuclear magnetic resonance study. Biochemistry, 28(21):8388-8396, 1989.

N. K. Sauter, J. E. Hanson, G. D. Glick, J. H. Brown, R. L. Crowther, S. J. Park, J. J. Skehel, and D. C. Wiley. Binding of influenza virus hemagglutinin to analogs of its cell-surface receptor, sialic acid: analysis by proton nuclear magnetic resonance spectroscopy and X-ray crystallography. Biochemistry, 31(40):9609-9621, 1992. 
M. J. Shlomchik and F. Weisel. Germinal center selection and the development of memory B and plasma cells. Immunological reviews, 247(1):52-63, 2012.

D. K. Takemoto, J. J. Skehel, and D. C. Wiley. A surface plasmon resonance assay for the binding of influenza virus hemagglutinin to its sialic acid receptor. Virology, 217(2):452458, 1996.

H. Taylor, S. Armstrong, and N. Dimmock. Quantitative relationships between an influenza virus and neutralizing antibody. Virology, 159(2):288-298, 1987.

C. M. Trombetta, E. J. Remarque, D. Mortier, and E. Montomoli. Comparison of hemagglutination inhibition, single radial hemolysis, virus neutralization assays, and ELISA to detect antibody levels against seasonal influenza viruses. Influenza and Other Respiratory Viruses, 12(6):675-686, 2018.

G. Tutz. Sequential models in categorical regression. Computational Statistics \& Data Analysis, 11(3):275-295, 1991.

D. Tyrrell and R. Valentine. The assay of influenza virus particles by haemagglutination and electron microscopy. Microbiology, 16(3):668-675, 1957.

P. A. Underwood. Problems and pitfalls with measurement of antibody affinity using solid phase binding in the ELISA. Journal of immunological methods, 164(1):119-130, 1993.

M. Von Smoluchowski. Mathematical theory of the kinetics of the coagulation of colloidal solutions. Z. Phys. Chem, 92(129):129-68, 1917.

E. G. Weinhold and J. R. Knowles. Design and evaluation of a tightly binding fluorescent ligand for influenza A hemagglutinin. Journal of the American Chemical Society, 114(24): 9270-9275, 1992.

WHO. WHO manual on animal influenza diagnosis and surveillance. 2002.

J. A. Williams, L. Gui, N. Hom, A. Mileant, and K. K. Lee. Dissection of epitope-specific mechanisms of neutralization of influenza virus by intact IgG and Fab fragments. Journal of Virology, 92(6):e02006-17, 2018.

I. A. Wilson, J. J. Skehel, and D. Wiley. Structure of the haemagglutinin membrane glycoprotein of influenza virus at 3 å resolution. Nature, 289(5796):366-373, 1981.

T. W. Yee et al. The VGAM package for categorical data analysis. Journal of Statistical Software, 32(10):1-34, 2010. 


\section{Supplementary Figures}

a

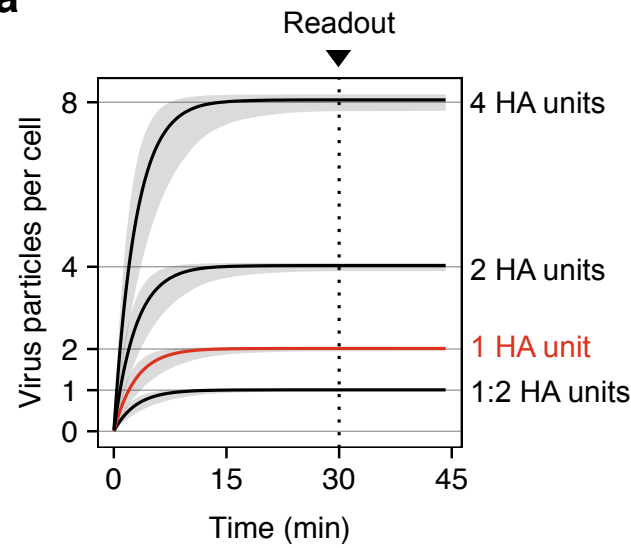

b

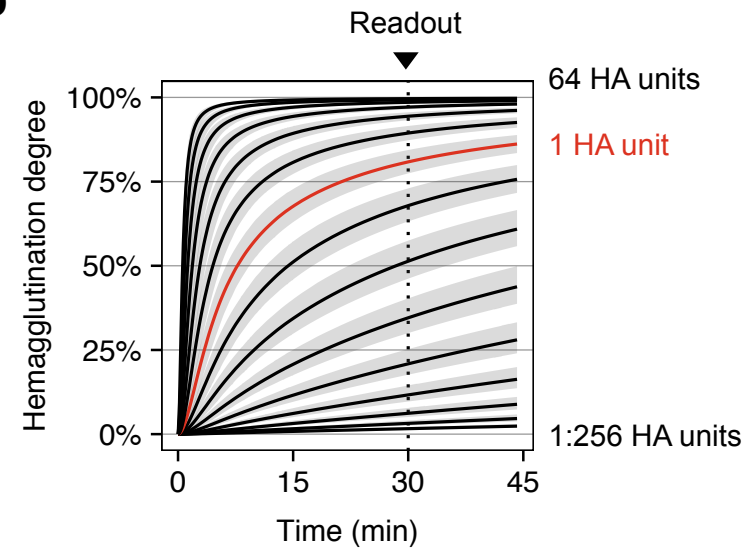

C

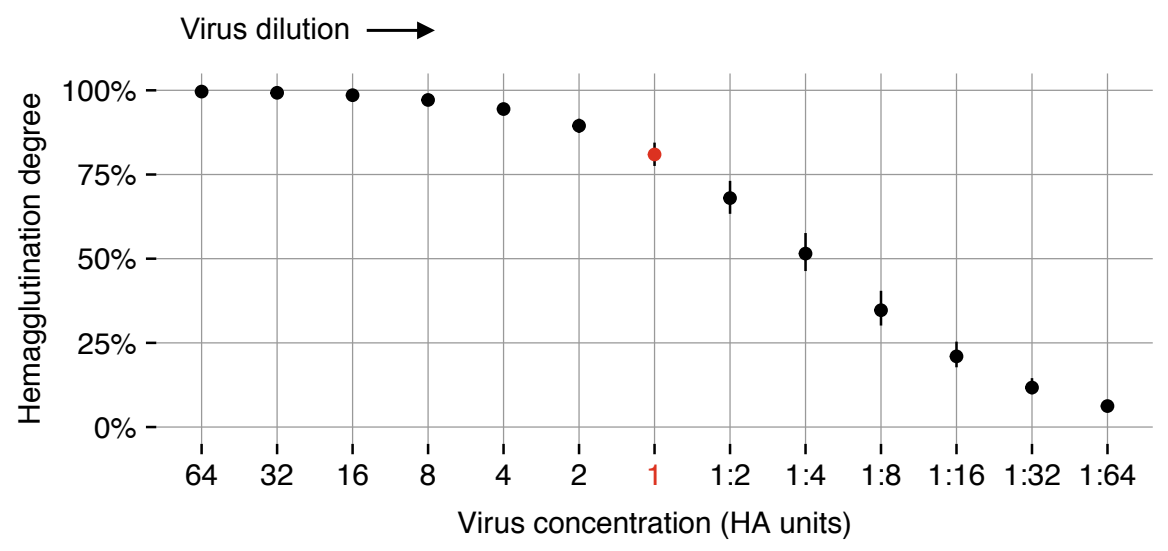

d

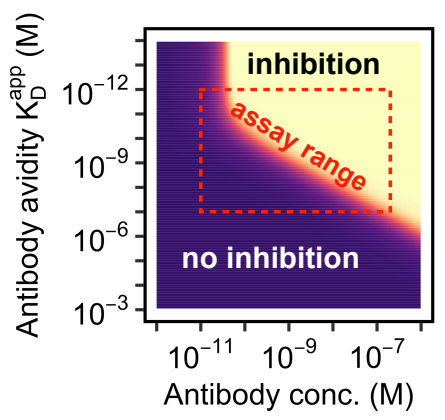

e

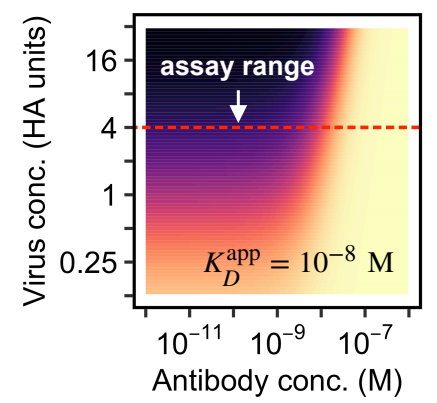

Hemagglutination degree

$\begin{array}{cc}\text { No } & -100 \% \\ \text { inhibition } & -75 \% \\ \text { Partial } & -50 \% \\ \text { inhibition } & 25 \% \\ \text { Inhibition } & 0 \%\end{array}$

Figure S1. Simulation results for the HA titration assay with influenza H1N1pdm09 and model sensitivity. (a) Binding kinetics of virus particles to red blood cells. We assume that full hemagglutination requires at least two bound virus particles per cell. (b) Hemagglutination kinetics. (c) For HA units $\geq 1$, the hemagglutination degree is $>75 \%$, which is by definition interpreted as full hemagglutination. Gray areas and error bars indicate the uncertainty due to uncertainty in model parameters. (d) Performing the HI assay with 4 HA units balances sensitivity and robustness. There is a clear distinction between inhibition and no inhibition. (e) At the same time, the assay detects with 4 HA units lower antibody concentrations than with $\geq 8$ HA units. 
bioRxiv preprint doi: https:/doi org/10.1101/202010 05.326215; this version posted February 23,2021 . The copyright holder for this preprint (which was not certified by peer review) is the author/funder, who has granted bioRxiv a license to display the preprint in perpetuity. It is made available under aCC-BY-NC-ND 4.0 International license.

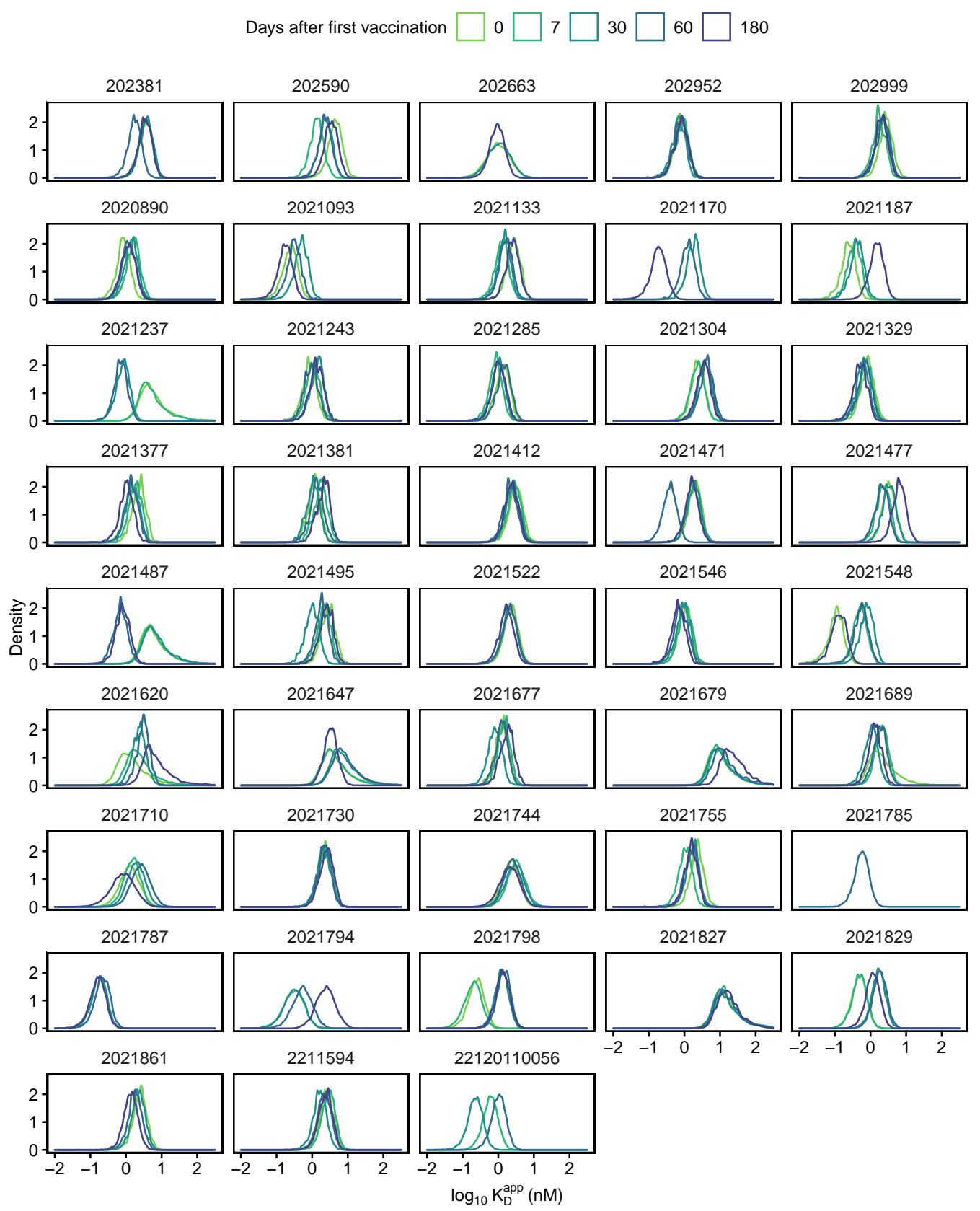

Figure S2. Marginal posterior distributions of apparent dissociation constants in 43 patients (197 posteriors in total). Some posteriors show larger variance due to larger measurement error in ELISAdetected IgG concentrations. 

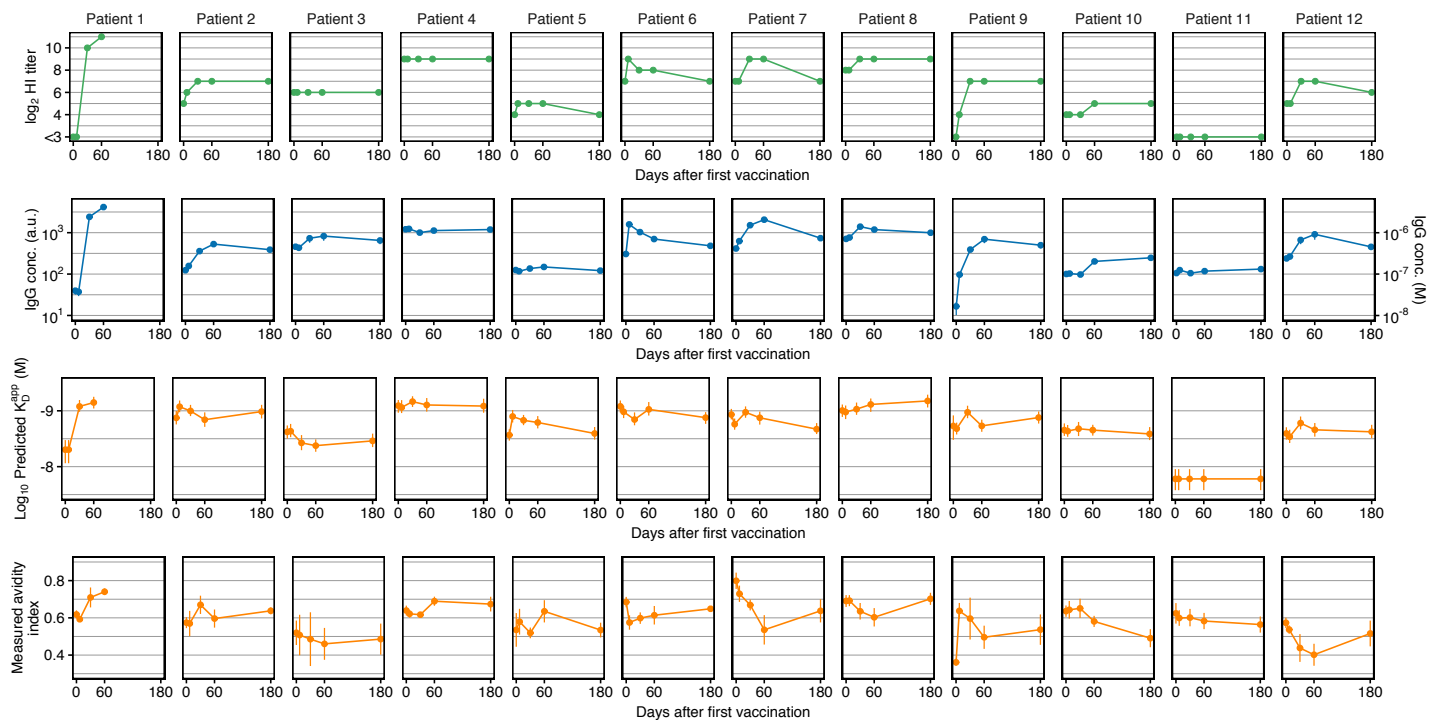

Figure S3. HI titer, ELISA-detected anti-H1N1pmd09 serum IgG concentration, inferred apparent dissociation constant and experimentally determined avidity index in twelve patients. Avidity indices correspond to the fraction of H1N1pmd09-specific serum IgG remaining bound after 4M urea treatment. Data shows mean and standard deviation for serum IgG and avidity indices and the maximum a posteriori (MAP) estimates and uncertainty range due to discretized HI titer measurements and ELISA measurement error for inferred dissociation constants $K_{\mathrm{D}}^{\mathrm{app}}$. Most patients showed either little or no increase in avidity. In some patients, the measured avidity decreased and then returned back to baseline on $\mathrm{d} 180$, potentially because vaccine-induced short-term antibodies were more sensitive to urea treatment, resulting in antibody denaturation. 


\section{a}

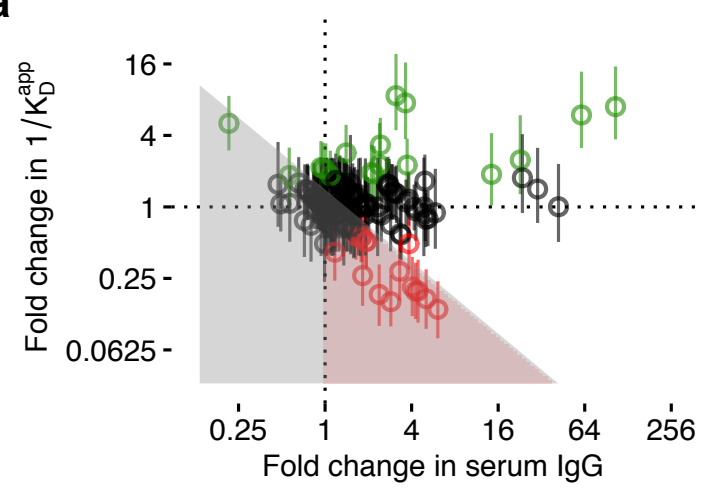

$\phi$ Increase in neutralizing serum avidity

$\phi$ No detectable change

$\phi$ Decrease in neutralizing serum avidity

Regions

No increase in $\mathrm{HI}$ titer

Increase in nonneutralizing $\lg G$

b

Fit results for avidity $1 / K_{D}^{\text {app }}$

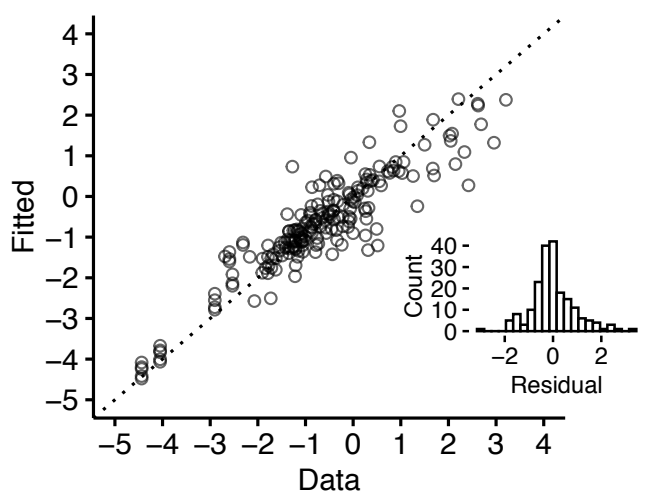

Fit results for serum IgG

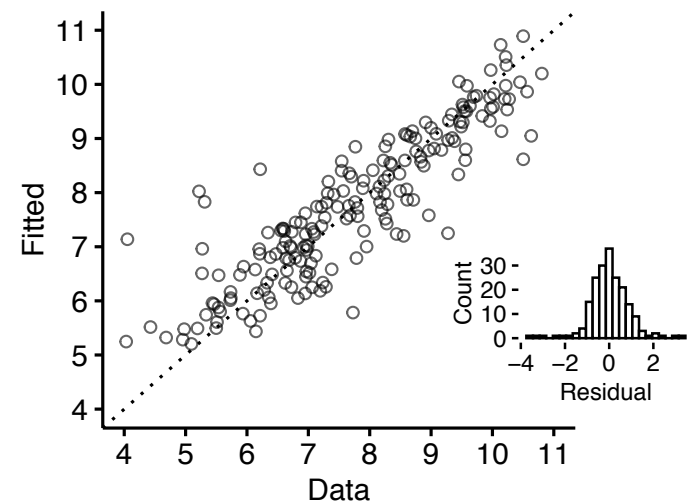

Figure S4. Vaccine response analysis in 43 patients (197 samples in total). (a) Fold change in inferred avidity and serum $\mathrm{IgG}$ after vaccination. Error bars indicate uncertainty in fold change due to uncertainty in inferred $K_{\mathrm{D}}^{\mathrm{app}}$-values. Shading indicates regions with qualitatively different responses to vaccination. (b) Tukey-Anscombe plot and histogram of the residuals of the regression models used to investigate differences in avidity and serum IgG. 

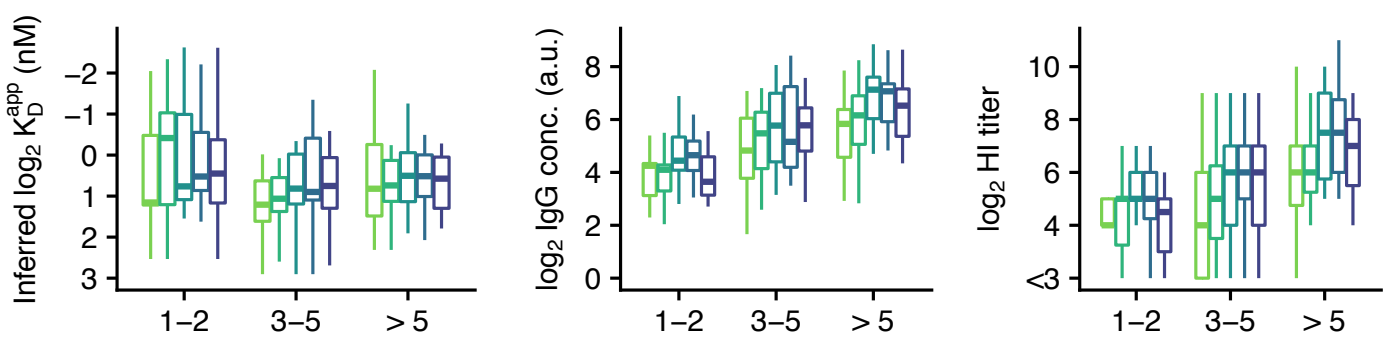

Time after transplantation (years)
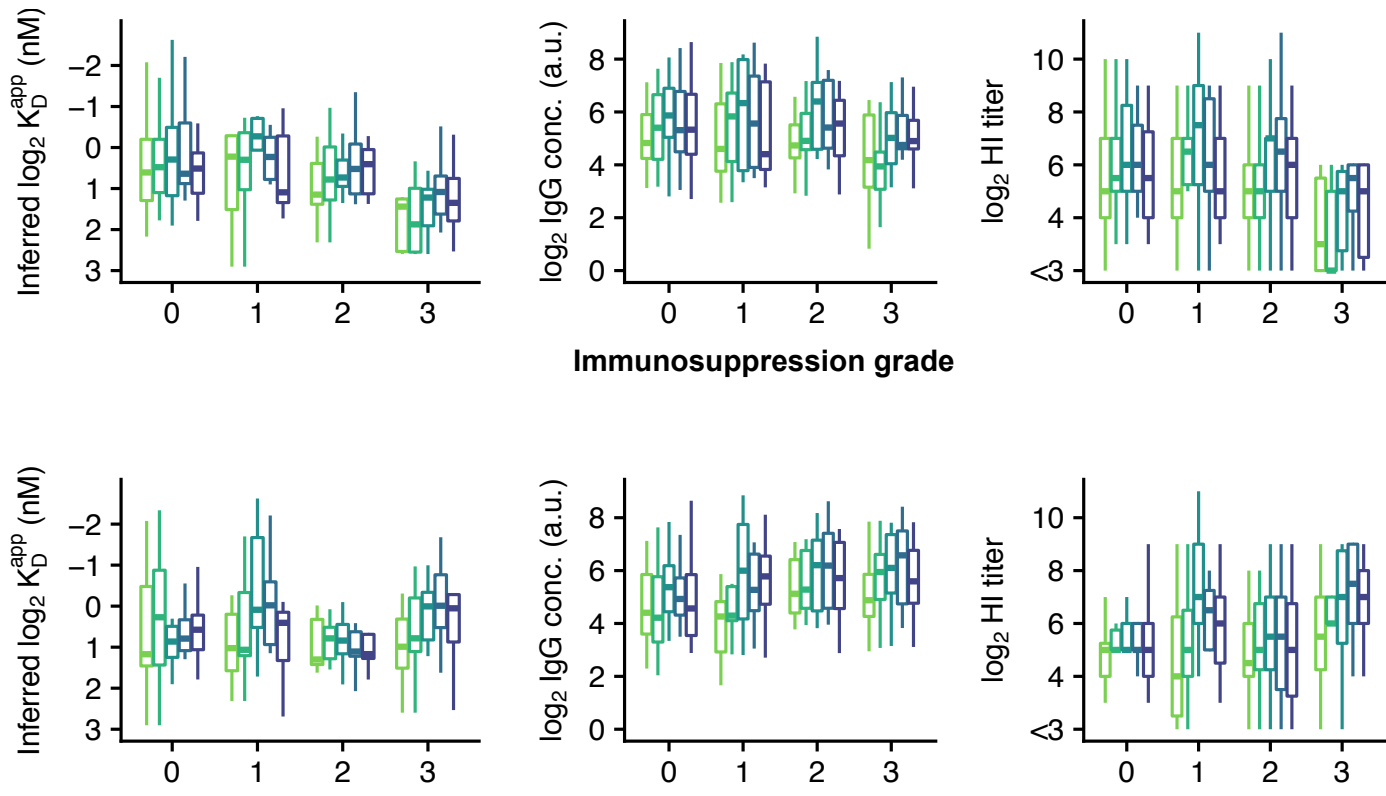

Chronic graft-versus-host disease grade

Figure S5. Inferred avidity, serum IgG concentration, and HI titers by time after transplantation, immunosuppression grade, and cGVHD grade in 43 patients (197 samples in total). Note that data show one-dimensional associations, whereas regression analysis was performed with a high-dimensional model simultaneously accounting for time after transplantation, immunosuppression/cGVHD grade, and correcting for sex and age. 